\title{
Time to Fly: A Comparison of Marginal Value Theorem Approximations in an Agent-based Model of Foraging Waterfowl
}

Matt L. Miller ${ }^{\mathrm{a}}$ (hzmiller@ucdavis.edu), Kevin M. Ringelman ${ }^{\mathrm{b}}$ (kringelman@agcenter.lsu.edu), John M. Eadie ${ }^{c}$ jmeadie@ucdavis.edu), and Jeffrey C. Schank ${ }^{a}$ (jcschank@ ucdavis.edu)

${ }^{a}$ University of California, Psychology Department, One Shields Ave., Davis, CA 95616

${ }^{\mathrm{b}}$ Louisiana State University, School of Renewable Natural Resources, LSU Agricultural Center, Baton Rouge, LA 70803

${ }^{\mathrm{c}}$ University of California, Wildlife, Fish, and Conservation Biology, One Shields Ave., Davis, CA 95616

Corresponding Author: Matt L. Miller, University of California, Psychology Department, One Shields Ave., Davis, CA 95616, hzmiller@ucdavis.edu, +1-530-752-6332, FAX +1-530-752-2087

(C) 2016. This manuscript version is made available under the Elsevier user license http://www.elsevier.com/open-access/userlicense/1.0/ 


\begin{abstract}
One of the fundamental decisions foragers face is how long an individual should remain in a given foraging location. Typical approaches to modeling this decision are based on the marginal value theorem. However, direct application of this theory would require omniscience regarding food availability. Even with complete knowledge of the environment, foraging with intraspecific competition requires resolution of simultaneous circular dependencies. In response to these issues in application, a number of approximating algorithms have been proposed, but it remains to be seen whether these algorithms are effective given a large number of foragers with realistic characteristics. We implemented several algorithms approximating marginal value foraging in a large-scale avian foraging model and compared the results. We found that a novel reinforcementlearning algorithm that includes cost of travel is the most effective algorithm that most closely approximates marginal value foraging theory and recreates depletion patterns observed in empirical studies.
\end{abstract}

\title{
Keywords
}

optimal foraging theory, decision-making, algorithm, simulation 


\section{$1 \quad 1 \quad$ Introduction}

Optimal patch selection describes how foragers should be distributed across

3 heterogeneous landscapes with respect to food items and to each other. Under simplifying

4 assumptions, optimality theory predicts that foragers consuming continually-replenishing

5 resources might select patches according to the ideal free distribution (Fretwell \& Lucas, 1969),

6 in which animals distribute themselves in the patches proportionate to the gain rate of resources.

7 However, for many animals, patch selection is dynamic: when resources are depleted in one area,

8 animals must find new patches containing those resources. In 1976, Charnov proposed marginal

9 value theorem (MVT), an analytical solution for determining when to leave a foraging patch that

10 predicts that a forager should depart when the intake rate for that patch falls below the long-term

11 average intake rate across all available patches. Charnov's theoretical result sparked a flurry of

12 empirical and modeling studies that suggested other patch-departure rules including fixed-time,

13 fixed-intake, minimizing, maximizing, and inter-reward intervals (e.g., Cowie, 1977; Hodges,

14 1981; Iwasa, Higashi, \& Yamamura, 1981; McNair, 1982; Green, 1984; McNamara \& Houston, 15 1985).

There remains some uncertainty as to how well any animal fits the predictions of MVT

17 and whether any animal could actually gather the information required to implement MVT

18 (Stephens \& Krebs, 1987; Stephens, Brown, \& Ydenburg, 2007). Nonetheless, animals likely use

19 some foraging strategy to be able to thrive in their environment, whether through evolution,

20 learning, development, or some combination of these processes. We may expect such strategies

21 to approximate MVT.

22 Since MVT has broad application, including agent-based models (ABMs) that explore

23 how individual behaviors scale up to create patterns at larger scales (Grimm et al., 2005; 
1 DeAngelis \& Mooij, 2005; McLane, Semeniuk, McDermid, \& Marceau, 2011) and non-

2 biological systems such as robot foraging (Ulam \& Balch, 2004), finding the best algorithmic

3 approximation for MVT is of critical importance for applied foraging contexts. In this paper, we

4 explore several avenues by which behavioral and biological researchers can optimally program

5 foraging agents that approximate MVT, using overwintering waterfowl as a test species. In

6 particular, we are interested in which algorithms provide the longest survival times in an

7 environment with depleting resources. We examine two approaches suggested in the robotics

8 literature, subsequently modified to account for travel time, and examine how these different

9 approximations to MVT affect energy intake and survival in our modeled system.

\subsection{Marginal Value Theorem}

11 We consider three different implementations of marginal value theorem in this paper:

12 Charnov's (1976) original MVT, or classical MVT; an algorithm approximating MVT based on

13 reinforcement learning (Wawerla \& Vaughan, 2009), or reinforcement-learning MVT; and an

14 algorithm approximating MVT using continuously-updated estimates (Wawerla \& Vaughan,

15 2010), or online MVT (abbreviations are summarized in Table 1). In the latter two cases, we

16 consider both the original techniques suggested by Wawerla and Vaughan as well as modified

17 versions of these methods that take into account differential costs of travel between foraging

18 areas, or extended reinforcement-learning MVT and extended online MVT. 


\begin{tabular}{ll}
\hline Abbreviation & \multicolumn{1}{c}{ Meaning } \\
\hline ABM & Agent-based model \\
DT50M & Days to 50\% mortality \\
DTD & Days to deficit \\
GUD & Giving-up density \\
IFD & Ideal free distribution \\
LTFA & Long-term-forward average \\
MVT & Marginal value theorem \\
OMVT & Online MVT algorithm \\
RLMVT & Reinforcement-learning MVT algorithm \\
XOMVT & Extended OMVT algorithm \\
XRLMVT & Extended RLMVT algorithm \\
\hline
\end{tabular}

Table 1. Abbreviations used in this paper.

\section{$1 \quad 1.2$ Classical MVT}

2 In 1976, Charnov proposed a mathematical model for the amount of time a forager

3 should remain in a patch of a given quality. If we consider that patches can be assigned a patch

4 type based on patch quality, identified by $p$, and a forager has a net gain in a patch of type $p$ of

$5 g_{p}\left(T_{p}\right)$ if it spends $T_{p}$ amount of time in that patch type, then the marginal rate of intake as length

6 of time in the patch type increases is

$$
\frac{\partial g_{p}\left(T_{p}\right)}{\partial T_{p}}
$$

7 (See Table 5 for notation). Similarly, if we are given the travel time between patches, $t$ ', and the

8 cost of travel $E_{\text {travel }}$, we can calculate the net intake rate for each patch type,

$$
\frac{g_{p}\left(T_{p}\right)-t^{\prime} E_{\text {travel }}}{t^{\prime}+T_{p}}
$$

9 If we know the proportion of each patch type in the environment, $\pi_{p}$, we can then calculate the

10 average net intake rate for the whole environment,

$$
\frac{\sum_{p} \pi_{p} g_{p}\left(T_{p}\right)-t^{\prime} E_{\text {travel }}}{t^{\prime}+\sum_{p} \pi_{p} T_{p}}
$$

11 Charnov showed that $T_{p}$ is optimized when the marginal rate was equal to the average net intake

12 rate for the environment. That is, more time spent in the patch would be wasted effort and less

13 time would fail to exploit useful resources. 
Calculating this rate requires omniscient knowledge of patch quality across the

2 environment. The gain function $g$ must be sufficiently characterized to calculate its rate of

3 change as time-in-patch increases, and for heterogeneous patches, $g$ must be characterized for all

4 patches in the environment. Even in the case of a single forager it is uncertain that the gain

5 function can be adequately characterized (see, for example, Stephens \& Krebs, 1987) because

6 average intake rate depends on the time in patches, and time in patches depends on average

7 intake rate. With multiple foragers and exploitation competition, both marginal and net intake

8 rates change as a function of the number of foragers in the patch; an ideal forager would not only

9 resolve its own circular dependency in MVT, but would also have to solve it for every other

10 forager. Ignoring the fact that animals cannot gather this information in the real world (Barton \&

11 Hovestadt, 2013), the problem of circularity in applying MVT has suggested that computation

12 even with perfect information is intractable (Wawerla \& Vaughan, 2010). These difficulties in

13 applying MVT are not surprising since MVT was derived as a theoretical optimum that behavior

14 might approach in the limit, not as a strategy for decision-making and not under real-world

15 conditions such as resource competition and depletion (Stephens \& Krebs, 1987; Wajnberg et al., 16 2006).

\section{$17 \quad 1.3 \quad$ Reinforcement-learning MVT}

18 Because of these difficulties in developing optimal foraging algorithms that satisfy MVT,

19 algorithms that approximate MVT have been proposed to determine time in patches, often

20 relying on very simple rules (Gibb, 1958; Krebs, 1973; Krebs, Ryan, \& Charnov, 1974;

21 McNamara, 1982). One of the more promising approaches was proposed by Wawerla and

22 Vaughan (2009) who estimated the optimal patch departure time by simulating reinforcement

23 learning, which we refer to as reinforcement learning MVT, or RLMVT.

24 RLMVT is based on an approach to simulating reinforcement learning by implementing 
1 the $n$-armed bandit using softmax to overcome local optima (Sutton and Barto, 1998; see

2 Supplemental Material C.1). This algorithm can be used to optimize foraging behavior. Consider

3 that the essential problem in implementing MVT is determining the energy gain rate at which the

4 agent should switch patches (the switching threshold). An agent can choose to switch at too high

5 a rate, which will result in spending too little time in any given patch; it can choose to switch at

6 too low a rate, which will result in spending too much time in any patch; or it can choose the

7 optimal rate, which should converge with the predictions of MVT. If we consider each of the

8 switching thresholds as one of the slot machine's $n$ arms and we equate the net gain across all

9 patches using that switching threshold as its reward, we can use the $n$-armed bandit algorithm to

10 find the optimal rate at which to switch patches under MVT. That is, the expected reward for a

11 given switching threshold (corresponding to an arm) is the average net gain rate experienced in

12 patches for which that threshold was used. The softmax algorithm helps greedy optimization

13 systems like the $n$-armed bandit from settling into local basins of attraction by making choices

14 that currently appear less than optimal more likely to be selected; it also prevents the expected

15 reward values for less-likely thresholds from becoming outdated by allowing them to be selected

16 occasionally (and thus updated) throughout the simulation. Softmax uses a parameter called

17 temperature $(\tau)$, in which higher temperatures make less-optimal-appearing choices increasingly

18 likely, going to equal probabilities for all choices regardless of expected reward at infinitely-high

19 temperatures (see Supplemental Material C.1 for softmax details).

20 There are two complications: (1) switching threshold is a continuous variable, while the

21 arms on the slot machine represent discrete values; and (2) the optimal switching threshold may

22 change as food resources are depleted. Wawerla and Vaughan (2009) conceptualized this

23 mapping between MVT and the $n$-armed bandit and addressed the first complication by 
1 generalizing Sutton and Barto's algorithm for a continuous action space (detailed in

2 Supplemental Material C.1). The second complication arises as a result of agents foraging within

3 a finite environment: classical MVT assumes an infinitely large landscape in which individual

4 patch depletion does not change the overall long-term average gain rate (Charnov, 1976). In real

5 environments, depletion over time continuously lowers the average gain rate if there is no

6 replenishment, and thus the optimal switching threshold also decreases. This can be accounted

7 for implicitly by setting $\tau$ to a reasonably high level, which increases the chances of testing

8 multiple switching thresholds regardless of expected reward.

9 Using RLMVT, a foraging agent can determine and track the switching threshold that

10 provides it with the highest long-term gain rate across encountered patches. The agent

11 accomplishes this empirically without categorizing patches, determining average densities across

12 the environment, or making explicit corrections for resource competition or depletion. The agent

13 simply forages in a patch until the gain rate drops to the level that the agent has learned will

14 optimize its overall gain if it switches patches.

\section{$15 \quad 1.4 \quad$ Online MVT}

16 Wawerla and Vaughan (2010) sought to improve their prior reinforcement-learning

17 technique with a continuous estimation algorithm we refer to as online MVT, or OMVT. The

18 authors hoped to overcome the requirement for startup initialization (during the first $n$ steps) and

19 inefficiencies of the softmax algorithm (see Supplemental Material C.1). To this end, they

20 devised a method that estimates the long-term average gain rate by continuously updating that

21 estimate from ongoing foraging experience (that is, online estimation).

22 Using OMVT, the foraging agent keeps track of the gain rate it experiences over time and

23 the time it takes the agent to switch patches on average. From this information, the agent

24 develops an estimate of the average gain rate across patches in its environment (see 
1 Supplemental Material C.2 for algorithm details). The agent compares its current gain rate to its

2 estimate of the average gain rate and switches to a new patch when the current rate falls below

3 the estimated average rate.

$4 \quad 1.5 \quad$ Extended MVT methods

5 The above algorithms above do not include the costs of traveling between patches; indeed,

6 travel cost is explicitly omitted in OMVT (Wawerla \& Vaughan, 2010). However, the

7 opportunity cost of travel time is still included by its effect on gain rate. In Wawerla and

8 Vaughan's research domain (addressed to robotics researchers), the energetic cost of traveling is

9 not accounted for as part of the foraging model since those robots were not foraging for energy.

10 However, in domains in which energetic costs must be minimized within a finite time horizon,

11 the cost of travel due to patch-switching decisions may become important, since any strategy that

12 increases how often an agent switches patches will ultimately lead to an increase in number of

13 switches per item retrieved, and thus increased cost for that strategy.

14 In animal foraging models, one must consider the cost of switching patches to obtain

15 more food; thus, the net energy gain that allows for the cost of traveling to a new patch must be

16 used to optimize foraging behavior. Furthermore, unlike robots, most foragers are limited in the

17 amount of foraging they can accomplish in one day due to limits in food intake and daily

18 behavioral patterns such as sleep. Because of these constraints, when resources are plentiful (for

19 example, early in the foraging season), foragers may not ever reach the optimal switching

20 threshold even in a single patch before they are done foraging for the day. When this is combined

21 with central-place foraging behavior, which requires the forager to return to a central location at

22 the end of a foraging bout then travel back to a foraging patch at the beginning of the next bout,

23 the energetic cost of switching patches may assume an even larger role in decision-making,

24 especially as patches become depleted. Since these constraints were not applicable to Wawerla 
1 and Vaughan's $(2009 ; 2010)$ research question, their algorithm was explicitly simplified to

2 exclude consideration of patch-switching cost.

3 However, as we are considering the behavior of animals like waterfowl, which are

4 central-place foragers with dietary and behavioral limits to foraging behavior, we extend

$5 \quad$ RLMVT and OMVT to include the cost of switching between patches.

6 The adjustments required are simple. For extended RLMVT (XRLMVT), we simply

7 include the cost of travel to the patch when calculating the achieved gain rate for each patch, $\mu$

8 (see Supplemental Material, Equation C3):

$$
\mu=\frac{f-t^{\prime} E_{\text {travel }}}{t^{\prime}+t},
$$

9 where $f$ is the amount of food obtained, $E_{\text {travel }}$ is the energetic cost of inter-patch travel, $t^{\prime}$ is the

10 time spent traveling to the patch, and $t$ is the amount of time spent foraging in the patch.

11 For extended OMVT (XOMVT), we adjust the total sum of previous gains, $G$, used to

12 calculate the estimated long-term gain rate (see Supplemental Material, Equation C11). In

13 OMVT, when the agent is in patch $p$,

$$
G=\sum_{q=1}^{p-1} g_{q}\left(T_{q}\right) .
$$

14 where $g_{q}\left(T_{q}\right)$ is the gain in patch $q$ when a forager spends time $T_{q}$ in that patch. For XOMVT, this

15 becomes

$$
G=\sum_{q=1}^{p-1} g_{q}\left(T_{q}\right)-t^{\prime}{ }_{q} E_{\text {travel }},
$$

16 where $t_{q}^{\prime}$ is the time taken to switch from patch $q-1$ to patch $q$.

17 Note the effects of these small changes on how each model behaves. In the case of

18 RLMVT, the extended model decreases the expected reward of a given switching threshold; thus,

19 high thresholds that lead to more frequent switching will be penalized. In the case of OMVT, the 
1 reduction in $G$, which is in the numerator of the estimated average gain rate, leads to a decrease

2 in that rate; thus, the forager will stay in the patch longer since lower rates of return will be

3 tolerated.

4 These slight modifications increase the biological validity of the model. We hypothesized

5 that they would also provide better decision-making for our agents, leading to more effective

6 foraging on a biologically-realistic landscape.

\section{Methods}

8 To assess the best decision-making algorithm, we operationalized foraging effectiveness

9 in two ways: food acquisition efficiency and survival time. Food acquisition efficiency was

10 assessed by days to deficit (DTD), the number of days elapsed before the average forager

11 expended more energy than it acquired (based on a three-day rolling average to prevent early

12 detection resulting from stochastic behavior). Survival time was assessed by the number of days

13 to $50 \%$ mortality (DT50M), when half the population had died due to starvation. DTD and

14 DT50M provided an absolute measure of the algorithm's effectiveness. In particular, longer

15 survival times (DT50M) given the same amount of food in the environment indicate more

16 efficient use of those resources.

17 Simulation of foraging was conducted using a pre-existing model of waterfowl foraging

18 behavior, SWAMP (Miller, Ringelman, Schank, \& Eadie, 2013). This agent-based model (ABM)

19 was created as a conservation management decision support tool for mixed wetland-agricultural

20 areas. The version of SWAMP used for this study minimized topological differences in the

21 foraging landscape to regularly arranged squares with varying total food densities on patches of

22 the same type, as required to test MVT behavioral rules. A brief synopsis of the model with

23 flowcharts is provided in Supplementary Material A. Key elements of the model are described 
1 below.

2 The ABM described by Miller and colleagues (2013) was augmented for this study with a

3 component that allowed each forager to make patch-switching decisions during foraging bouts

4 using one of the MVT approximations listed above, either RLMVT, OMVT, XRLMVT, or

5 XOMVT. When this component indicated that the forager should switch patches, the forager

6 randomly picked one of the nearest patches to the current patch and moved to it. A foraging bout

7 encompassed all of the temporally-contiguous foraging behavior for a single day (that is, there

8 was effectively only one bout per day) regardless of the number of patches visited; foraging

9 bouts were delimited by return to the forager's home refuge. Patches that the forager had already

10 visited during the current foraging bout were excluded from consideration for switching until a

11 later bout (that is, the forager had to return to its home refuge before reconsidering a patch that

12 had already been visited).

13 For both reinforcement learning approximations (RLMVT and XRLMVT), all fixed

14 parameters in the $n$-armed bandit with softmax apparatus were held equal: foragers used 5 bins

15 ( $n$ in the equations above) and the softmax temperature was $15.0(\tau)$. Other parameters not

16 covered in the main text are described in the online supplement (Appendix A).

17 In addition to the MVT approximations, two alternatives were used as bracketing

18 comparisons. In the no optimization condition, foragers stayed in the patch to which they were

19 randomly assigned at the beginning of a foraging bout for the duration of that bout (the No

$20 \mathrm{MVT} /$ Random condition). Assignment was proportional to patch area; since patch areas were

21 equal in this model, each patch had an approximately equal number of foragers assigned to it at

22 the beginning of the foraging bout. In the ideal free distribution condition, foragers also

23 remained within the same patch throughout a given foraging bout; however, foragers were 
1 assigned to patches at the beginning of a bout using optimal matching based on the ideal free

2 distribution (IFD) theory (the No MVT/IFD condition). IFD, first proposed by Fretwell and

3 Lucas (1969), suggests that foragers will distribute themselves to match available resources so

4 that depletion due to foragers will be proportional to food availability. In this way, denser food

5 patches will deplete more rapidly so that patches of all qualities will be effectively exhausted at

6 the same time despite their original differences in food availability (Sutherland, 1996, p. 36-37).

7 Like classical MVT, however, IFD requires that foragers be omniscient in regards to the

8 distribution of food in their environment and the behavior of other foragers, a biologically

9 unreasonable assumption (Pierce \& Ollason, 1987). (IFD also has other limitations, e.g.,

10 Bernstein et al., 1988 and Bernstein et al., 1991, though these limitations do not affect its

11 usefulness as a comparison technique in these simulations.) Unlike MVT, IFD is computationally

12 tractable and thus serves as a reasonable model with which to compare the various algorithms.

13 We simulate IFD by stochastically distributing foragers proportional to the current food density

14 on patches at the beginning of each foraging bout; that is, in IFD simulations, agent patch

15 selection is optimized with respect to resources and other foragers on a daily basis.

16 A total of 80,000 agents were modeled on a simulated $10 \mathrm{~km}$ by $10 \mathrm{~km}$ landscape; the

17 number of agents was based on scaling the target ecosystem (see below) down to the modeled

18 area. Simulated time steps were a maximum of 15 minutes. Food availability on the landscape

19 was varied systematically, starting at maximum values replicating a well-studied waterfowl

20 foraging ecosystem, California's Central Valley (Central Valley Joint Venture, 2006): forageable

21 landscape at $35 \%$ of the landscape, composed of $80 \%$ commercial rice field and $20 \%$ moist soil

22 wetland habitat. We varied these proportions to produce foraging landscapes of different

23 abundance (see Table 2 for quantitites). These landscape variations were tested to determine if 
1 the comparative efficacy of foraging strategies differed in more or less abundant landscapes.

2 Simulations ended when all agents ran out of energy reserves.

\begin{tabular}{rrr}
\hline Moist Soil Acreage (ha) & Rice Field Acreage (ha) & Total Forageable Acreage (ha) \\
\hline 700 & 2700 & 3400 \\
1400 & 2000 & 3400 \\
2000 & 1400 & 3400 \\
400 & 1600 & 2000 \\
800 & 1200 & 2000 \\
1200 & 800 & 2000 \\
\hline
\end{tabular}

Table 2. Foraging acreage scenarios.

3 Basic parameters for agent metabolism, foraging efficiency, food energy values, and

4 patch food initial densities were held equal across all simulations at values previously found to

5 be biologically plausible (Miller et al., 2013). We present all model parameters in Appendix A

6 (Supplementary Material). Thirty trials were simulated for each combination of landscape

7 scenario, initial patch distribution (random or IFD), and MVT strategy (no patch switching,

8 RLMVT, OMVT, XRLMVT, and XOMVT). IFD was only used with no patch-switching strategy.

9 The basic premise of MVT is that optimal foragers should switch patches when their gain

10 rate falls below the long-term average gain rate in the environment; that is, the switching

11 threshold should be equal to the long-term average. Determining the long-term average would

12 allow a useful comparison of each algorithm's performance to actual MVT-predicted

13 optimization. However, as noted above, this average rate cannot be determined a priori because

14 of the circular causality between the average rate and forager behavior. Furthermore, classic

15 MVT considers the average rate to be a constant because it considers an infinite landscape in

16 which the long-term average rate across the landscape is unaffected by the depletion of

17 individual patches. This clearly is not the case in many ecosystems and was not the case in our

18 simulation. Real-world landscapes may only produce a fixed quantity of resources each season

19 which are exhausted before being replenished. Our simulations model this kind of landscape: as 
1 time moves forward, the agents have removed food resources so that patch densities decrease

2 and overall gain rates must therefore be lower than they were earlier in the simulation. Despite

3 our inability to calculate a long-term average gain rate in advance, it is possible to calculate the

4 achieved average gain after a simulation is complete. To account for the change in the overall

5 landscape's gain rate in finite time, instead of using a constant value for the average rate, we

6 calculated an average rate that changed on a daily basis to reflect resource depletion. This rate

7 was the average of daily gain rates only from the beginning of the given day through the end of

8 the simulation. A rate calculated in this way is affected by the foraging strategy in use, but

9 nonetheless does reflect the only true measurement possible of the rate in the environment, in

10 particular given that the environment includes the foraging behavior of its denizens. Because this

11 was the long-term gain rate going forward, we call this the long-term-forward average rate

12 (LTFA rate). Since a single day is short compared to the length of the overall simulation and

13 resource depletion across a single day is therefore relatively small compared to the total amount

14 of food in the landscape, the LTFA rate may be a reasonable approximation of the optimal

15 switching threshold for that day. Therefore, we used the LTFA rate as a metric to compare for the

16 achieved switching threshold to MVT predictions as a further test of the effectiveness of each

17 algorithm.

18 The ABM used herein collects additional data that the original model (Miller et al., 2013)

19 did not collect. The ABM used for this study calculated the mean and standard deviation of patch

20 density, the number of foragers in each patch, and the correlation between number of foragers in

21 a patch and that patch's density at each time step. This ABM also captured on a daily basis the

22 average gain rate for all foragers, the average gain rate at which foragers switched patches (that

23 is, the current average switching threshold), and the average number of patch switches per day. 
All statistical analyses were completed in R (R Core Team, 2013), including the

2 calculation of LTFA.

\section{$3 \quad 3 \quad$ Results}

For all analyses, we examined each landscape combination separately. However, we

5 noted that these results fell into two categories. In the first set of cases, all of the results showed

6 very similar patterns, simply scaled to the amount of food in the landscape (for example, a larger

7 number of simulation days for environments with more food). In the second set of cases, there

8 was a continuum of results from the least rich environment to the most rich environment. Thus,

9 for results belonging to the first set, we only present results from the richest environment (1400

10 ha rice, 2000 ha moist soil), and for results belonging to the second set, we present results from

11 the richest environment (as above) and the poorest environment (1600 ha rice, 400 ha moist soil).

12 Furthermore, OMVT and XOMVT results are very similar with slight scaling differences. Thus,

13 except for the direct measure of the outcomes of interest in which we report all conditions, we

14 report only the XOMVT results since these results were a slight improvement over OMVT.

15 Complete sets of graphs for all conditions and all strategies are presented in the supplementary

16 material, Appendix B.

17 One of the predictions of IFD is that patches with more resources will be depleted at a

18 faster rate than those with less resources so that patches will become more homogeneous as they

19 approach depletion; this pattern is observed in empirical observations of foraging environments

20 (Sutherland, 1996; Nolet et al., 2006). Typical results across simulation time are shown in Figure

211 for random patch selection with no MVT (Figure 1a), IFD with no MVT (Figure 1b), random

22 patch selection with RLMVT (Figure 1c), random patch selection with XRLMVT (Figure 1d),

23 and random patch selection with XOMVT (Figure 1e; OMVT is omitted as it performed 
1 similarly to XOMVT). Random and IFD both had very smooth progressions to their end states.

2 Random patch selection with no MVT strategy has uniformly decreasing patch density but

3 patches were not of uniform density at the end of the simulation; approximately half the patches

4 were depleted to the forager's giving-up density (GUD; the level below which they would no

5 longer attempt to utilize a patch), but many of the initially dense patches were not completely

6 exploited by the end of the simulation. IFD patch selection showed the expected pattern in which

7 more dense patches were exploited more than less dense patches; all patches reached a uniform

8 level near the GUD. RLMVT recapitulated the IFD results very closely, though with some

9 noisiness in the order in which patches were exploited; this raised the question, examined below,

10 of why RLMVT did not perform as well as IFD. XRLMVT appeared to be performing in much

11 the same way as random patch selection for most of the simulation, with early signs of

12 approaching IFD results at $75 \%$ of simulation time; by the end of the simulation, however,

13 XRLMVT also achieved efficient patch exploitation. This time course resulted from the patch-

14 switching algorithm being conservative in switching patches early in the simulation because of

15 the costs of moving and then becoming more willing to switch patches as patch densities became

16 very small. This allowed XRLMVT to obtain similar final results to IFD without incurring

17 switching costs when resources were plentiful. Both XOMVT and OMVT exploited patches in a

18 similar pattern to IFD, but at much later stages of the simulation than IFD and RLMVT (for

19 example, XOMVT's pattern at simulation end resembled RLMVT's at 75\%). Both also had

20 somewhat noisier exploitation patterns than the other strategies. This resulted from the large

21 number of patch switches early in the simulation while resources were still plentiful; this

22 depleted forager energy reserves unnecessarily and prevented them from completing efficient

23 utilization of the environment, as we examine next. 


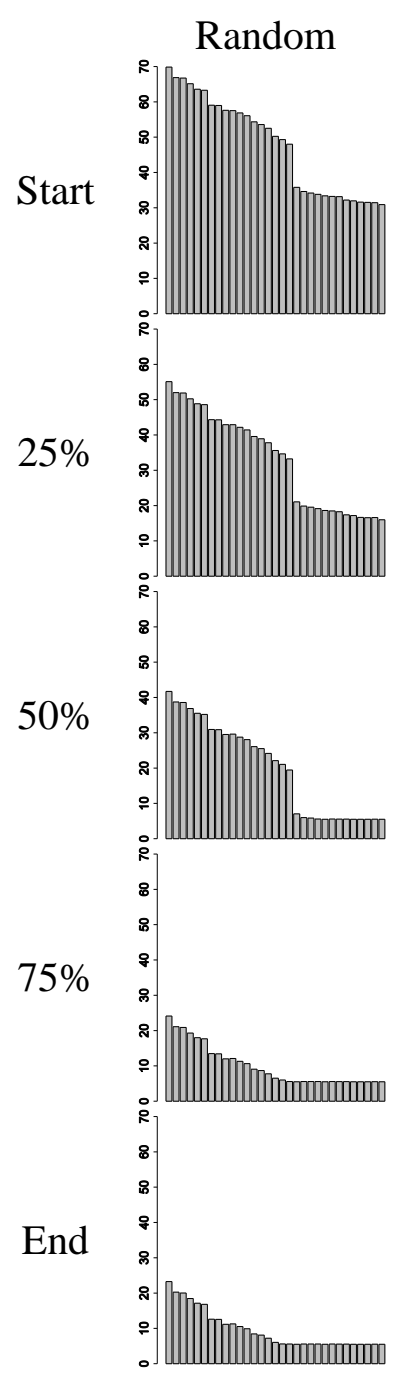

a
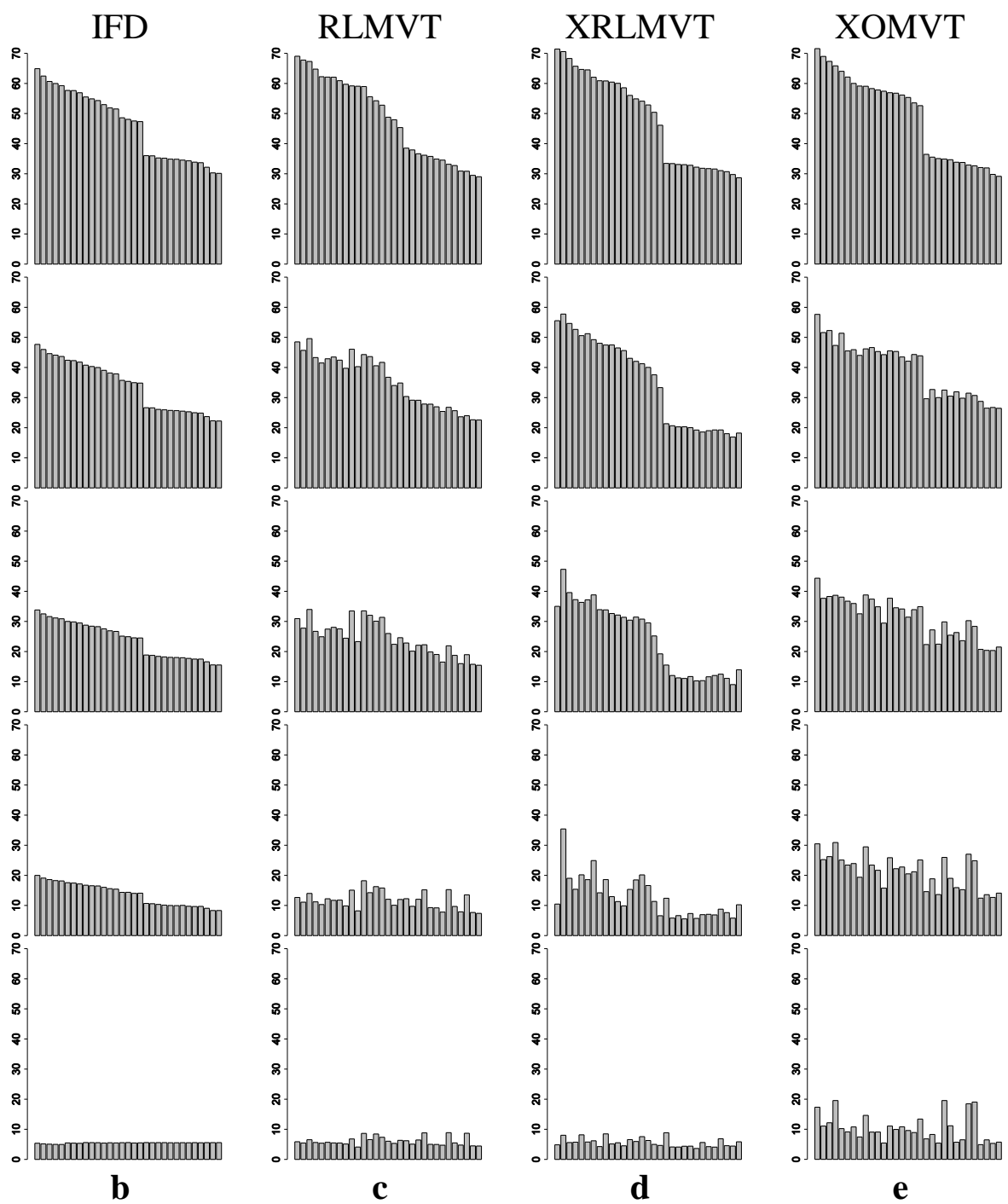

Figure 1. Patch density distributions over time for typical simulations: (a) random initial selection with no MVT, (b) IFD initial selection with no MVT, (c) random initial selection with RLMVT, (d) random initial selection with XRLMVT, and (e) random initial selection with XOMVT. All simulations shown above are for 1400 ha rice acreage and 2000 ha moist soil acreage. On all graphs, patches are consistently ordered by food density at the beginning of the simulation (that is, patch order on the $\mathrm{x}$-axis remains the same for all five time points graphed).

To determine energy expenditures for the foraging strategies, we analyzed the average

2 number of patch switches per foraging bout per forager for each, averaged across all 30 trials

3 (Figure 2). The XOMVT strategy, in particular, had an extremely high number of patch switches.

4 Before day 100, the XOMVT strategy causes foragers to switch more often than the maximum

5 number for RLMVT; before day 30, XOMVT leads to more switches than the maximum number

6 for XRLMVT. Ultimately, the XOMVT strategy peaks at more than four times as many switches 
1 per foraging bout as RLMVT and XRLMVT. Furthermore, XRLMVT peaks at only about half

2 the number of switches as RLMVT. This peak also occurs some 50 days later than the RLMVT

3 peak. This supports the idea that foragers using XRLMVT do not switch patches until very late

4 in the simulation, conserving resources needed for survival until efficient patch use becomes

5 necessary. The area under each curve can be thought of as a proxy for time spent switching

6 patches; direct examination of the Figure 2 should make it clear that XRLMVT foragers spend

7 substantially less time switching patches (particularly than XOMVT foragers), and consequently

8 spend substantially less energy switching patches.

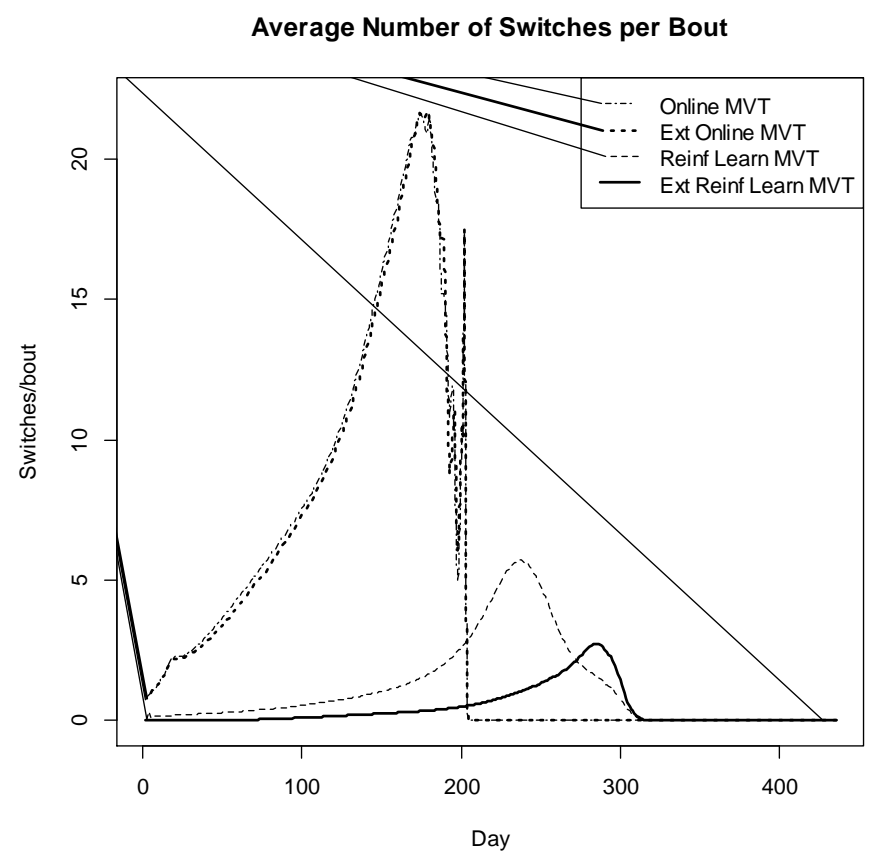

Figure 2 . Average switches per bout per forager for MVT approximation strategies (richest landscape condition).

To understand the basic MVT processes driving these results, we compared the achieved

11 gain rate, the gain rate at which agents switched patches (the switching threshold), and the LTFA

12 rate; the average number of switches per bout was graphed on the second axis to allow direct

13 examination of its relationship to the foraging metrics (Figure 3). Recall that our expectation is 
1 that the switching threshold (solid black line) should approach the LTFA rate (dashed red line)

2 when the forager is behaving in accordance with MVT. It is immediately obvious that in the

3 online MVT strategy (XOMVT) the switching threshold rapidly converges to the current overall

4 gain rate (solid red line), well above the LTFA rate (and for most of the first half of the

5 simulation, also greater than even the long-term gain rate at the beginning of the simulation). By

6 comparison, RLMVT does a fairly good job of tracking the LTFA rate, though in the poorest

7 landscape it overestimates the optimum switching threshold for the first half of the simulation.

8 XRLMVT performs most accurately in tracking the LTFA rate, almost exactly matching it in the

9 poorest landscapes. In richer landscapes, XRLMVT even slightly underestimates the LTFA rate,

10 which may be a preferable strategy if at the beginning of the simulation most patches are

11 sufficiently rich to provide for the agent's daily needs. In such cases, it may be unnecessary to

12 switch patches early on, until patch densities begin to fall so low that meeting daily energy

13 requirements becomes uncertain; therefore, foragers should avoid switching patches until later in 14 the simulation.

15 It is worth observing that the LTFA rates are not equal across the different algorithms. As

16 noted above, this rate is affected by the foraging strategies in use by the environments' foragers.

17 In particular, we draw attention to the fact that the XOMVT algorithm's LTFA rate is highest, the

18 RLMVT LTFA rate is lowest, and the XRLMVT has a LTFA rate intermediate between the two.

19 We examine this result in detail in the discussion, below. 

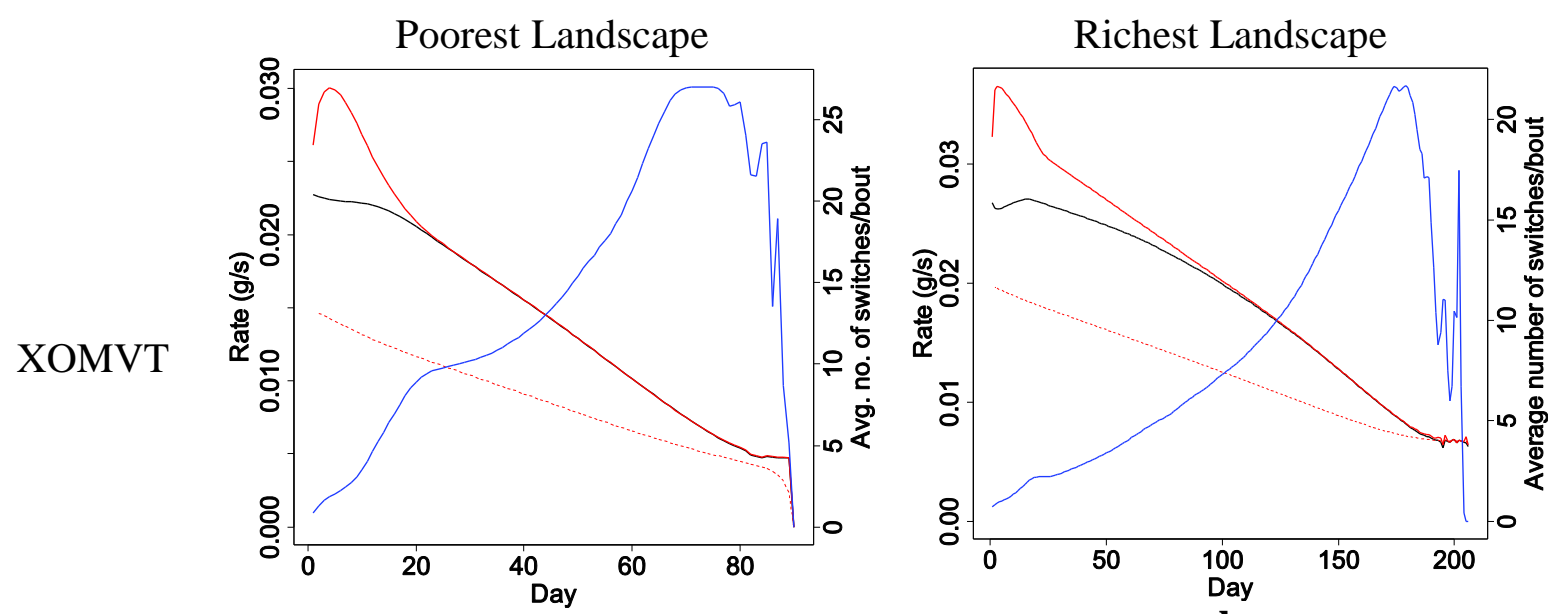

a
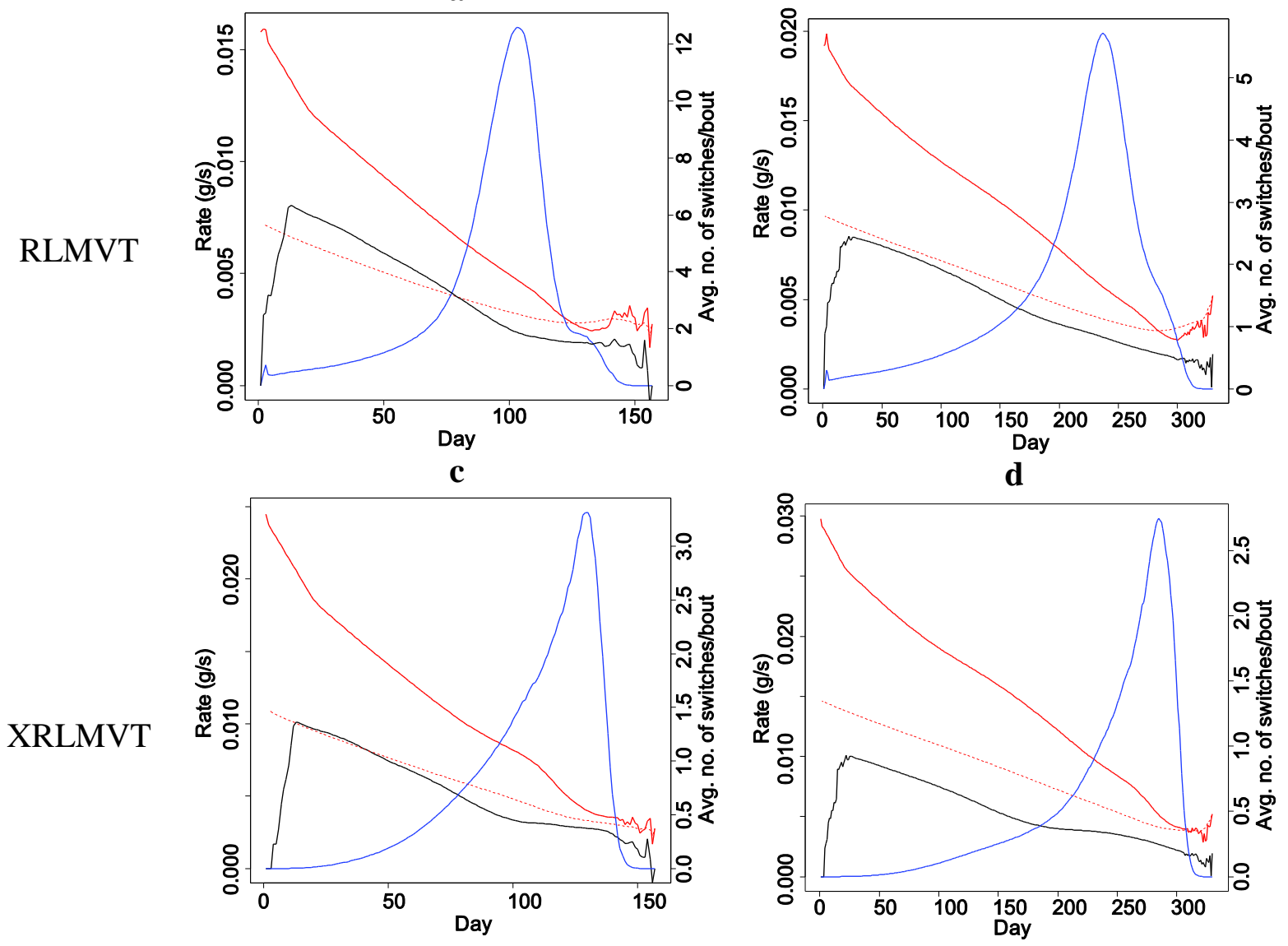

e

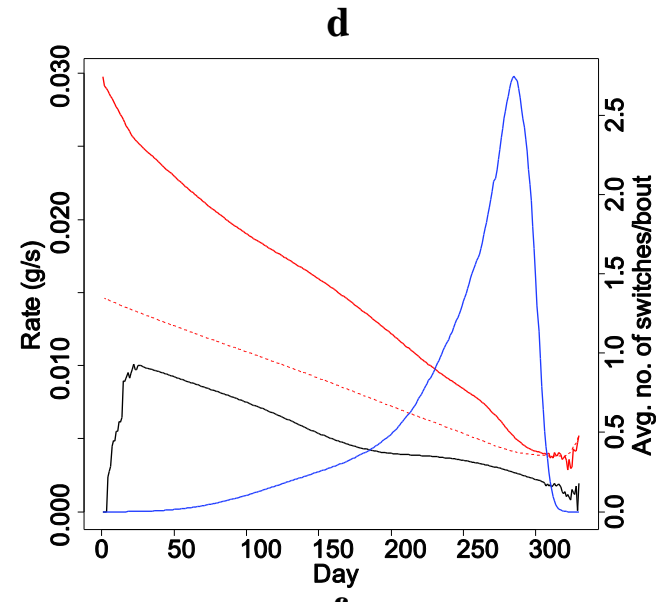

Figure 3. Overall average gain rate per forager (solid red line), LTFA rate (dashed red line), rate at patch switch (black line), and switches per bout per forager (blue line, on right-hand axis). XOMVT (a) and (b); RLMVT (c) and (d); and XRLMVT (e) and (f) are shown for poorest landscape conditions (a), (c), and (e); and for richest landscape conditions (b), (d), and (f). Note that scales change between graphs.

These processes led to the following results in our primary outcomes of interest. As

2 expected, the IFD simulations performed best by both measures of effectiveness. The order of 
1 effectiveness for the remaining conditions (again, for both measures, which were highly

2 correlated) from best performance to worst was XRLMVT, RLMVT, random patch assignment,

3 XOMVT, and OMVT (Tables 3 and 4).

4

\begin{tabular}{lrrrrr}
\hline & No MVT & OMVT & RLMVT & XOMVT & \multicolumn{1}{c}{ XRLMVT } \\
\hline IFD & 195.9 & & & & \\
\multirow{4}{*}{ Random } & $(4.65)$ & & & & \\
& 151.3 & 87.5 & 152.6 & 88.4 & 175.7 \\
& $(3.99)$ & $(2.38)$ & $(3.52)$ & $(2.46)$ & $(4.26)$ \\
\hline
\end{tabular}

Table 3. Days to deficit (DTD) for each condition (average across replications, standard errors in parentheses).

\begin{tabular}{lrrrrr}
\hline & No MVT & OMVT & RLMVT & XOMVT & XRLMVT \\
\hline IFD & 221.2 & & & & \\
\multirow{4}{*}{ Random } & $(4.62)$ & & & & \\
& 189.6 & 129.9 & 187.7 & 130.8 & 208.3 \\
& $(3.71)$ & $(2.63)$ & $(4.08)$ & $(2.72)$ & $(4.31)$ \\
\hline
\end{tabular}

Table 4. Days to $50 \%$ mortality (DT50M) for each condition (average across replications, standard errors in parentheses).

We determined each strategy's effect on the outcome variables by examining the

6 differences between conditions. Algorithms' improvements were judged in comparison to the

7 non-IFD, no-MVT results. XRLMVT improved both DTD and DT50M by the greatest amount

8 among MVT strategies (DTD, 24.32; DT50M, 18.69), though, as expected, this improvement

9 was not as great as that for IFD (DTD, 44.60; DT50M, 31.60). RLMVT did not change DTD or

10 DT50M by a substantial number of days (DTD, 1.28; DT50M, -1.96); indeed, for both measures,

11 the results are within the 95\% confidence intervals of the non-IFD, no-MVT algorithm. Both

12 OMVT and XOMVT resulted in decreases in DTD and DT50M suggesting once again that the

13 online MVT strategies result in excessive energy expenditure under all conditions.

\section{Discussion}

15 Our goal was to explore the effectiveness of several optimal foraging algorithms designed 
1 to approximate MVT. In our simulations of biologically-plausible foragers, extended versions of

2 Wawerla and Vaughn's algorithms $(2009 ; 2010)$ performed better across a range of landscape

3 conditions. However, we also found that for our simulation conditions and agents, reinforcement

4 learning algorithms (RLMVT and XRLMVT) performed far better than continuous estimation,

5 or online, algorithms (OMVT and XOMVT). Indeed, the online algorithms performed worse

6 than simple random patch assignment with no patch switching, while the reinforcement learning

7 algorithms approached the performance of IFD simulations. XRLMVT achieved an effectiveness,

8 compared to IFD simulations, of $89.7 \%$ for days to energy deficit and $94.2 \%$ for days to $50 \%$

9 mortality.

The factor that drives the differences in foraging effectiveness is the number of patch

11 switches that each algorithm imposes on foraging agents. The online algorithms are aggressive in

12 seeking better patches, and in so doing exhaust food resources and energy reserves very rapidly.

13 The reinforcement learning algorithms impose very low patch-switching rates until late in the

14 simulation, as patches become depleted, and thus conserve food and energy. Online algorithms

15 attempt to derive a switching threshold based on the agents' experiences of gain rates, while

16 reinforcement learning algorithms use achieved energy gains to determine if a threshold is more

17 or less useful. The online methods' divergences from foraging gains, focusing only on meeting

18 rate targets, drive their inefficiency. On the other hand, reinforcement learning algorithms select

19 any good-enough gains that minimizes energy expenditure, resulting in more conservative

20 switching until some patches are too depleted to provide net energy gains.

21 For systems in which short-term gain rate maximization is more important than length of

22 survival in a depleting landscape, however, our results suggest that the very dynamics that make

23 the online algorithms less effective under this study's assumptions would likely favor the online 
1 algorithms. Future research might also be conducted in which the various algorithms compete

2 against one another in the same environment to determine if the reinforcement-learning

3 algorithms' long-term survival and efficient use of resources are more evolutionarily beneficial

4 than the short-term acquisitiveness of the online algorithms, or if the converse is true in spite of

5 the online algorithms' shorter survival times. However, this question was beyond the scope of the

6 research reported herein.

$7 \quad$ Evidence that long-term efficiency drives the success of reinforcement-learning

8 algorithms in this study can be seen by comparing each algorithm's switching threshold with the

9 overall achieved gain rate and the LTFA rate. The online algorithms' switching threshold

10 converged to the achieved gain rate, which was well above any long-term gain rate average for

11 the environment, and thus not in line with classical MVT. On the other hand, the reinforcement

12 learning algorithms approached the LTFA rate for the simulated environments. Because the

13 reinforcement learning algorithms achieved high degrees of foraging effectiveness, we conclude

14 that the LTFA is an appropriate target threshold for patch switching in finite landscapes with

15 depletion. As we have discussed, the LTFA is definitely affected by the algorithm in use.

16 However, the online algorithms switching thresholds exceeded their LTFA rates despite the fact

17 that those algorithms had the highest LTFA rates; besides being a violation in the strict sense of

18 the basic idea of MVT (switching threshold should match the average gain rate), it could be

19 argued that these gain rates were inflated by the aggressive resource exploitation of that

20 algorithm. More surprisingly, even though XRLMVT was not as aggressive as the RLMVT

21 algorithm, it actually achieved higher LTFA rates. Therefore, despite the fact that LTFA is

22 dependent on the foraging strategies being used, thereby being somewhat circular itself, we argue

23 that this study demonstrates that it is the proper target to achieve in depleting finite landscapes. 
Further research into the XRLMVT algorithm would yield information regarding specific

2 parameterization of the reinforcement-learning system. In particular, adjustment of the softmax

3 temperature may improve foraging effectiveness. Since lower softmax temperatures favor

4 efficient exploitation of apparent optimal behaviors at the cost of exploration, but higher

5 temperatures favor exploration of changing conditions and alternative maxima at the expense of

6 efficient use of discovered optimal behaviors, the temperature parameter space should be

7 explored to find the optimal tradeoff value for these two competing forces. Alternatively,

8 annealing, in which the temperature is decreased as the simulation proceeds, or reverse-annealing,

9 in which the temperature is increased as the simulation proceeds, might be explored. Adjusting

10 the temperature in response to current forager success may also yield fruitful results: for instance,

11 lowering the temperature to exploit discovered optima when the cost of switching patches is

12 much lower than intake, but allowing the temperature to rise again as the cost of switching

13 patches begins to approach intake levels.

14 The research reported herein only models exploitative competition. Further research

15 regarding the effects of interference competition on these algorithms can be accomplished by

16 adding interference abilities to the agents. We expect that the additional patch switching imposed

17 by competition will increase cumulative travel costs (Fretwell, 1972), resulting in a decrease in

18 the necessary gain rate to remain in a patch. That would reduce the number of patch switches due

19 to patch quality. Such a result would be in accord with theoretical expectations, since under

20 interference competition it is more desirable for a forager to remain in a patch that it controls.

21 Since, as noted at the end of the description of RLMVT (above) our algorithms do not impose

22 assumptions about how gain rates emerge but allow foragers to simply maximize total gain based

23 on foraging experience, we predict that results with interference competition will be substantially 
1 similar to those in this study. This will have to be tested in future research. For our current report,

2 we note that there are many systems in which exploitative competition is more important than

3 interference competition, including the overwintering waterfowl that we used as our example

4 organism. Nevertheless, other factors such as predation risk (Sih, 1995; Sih, 1998), especially

5 from waterfowl hunters (Dooley et al., 2010; Lancaster et al., 2015), and nutritional requirements

6 (Loesch \& Kaminski, 1989) may influence patch selection and departure decisions.

$7 \quad$ Effective, efficient foraging strategies such as XRLMVT may find application in many

8 problems beyond ecological simulations. Specifically, since this algorithm performs optimally by

9 taking the cost of switching patches into account, XRLMVT might be well-suited to applications

10 that are particularly sensitive to movement costs. Models of mate choice, in which switching rate

11 must be calibrated for efficient evaluation of mates (Sullivan, 1994); models of group selection

12 and formation (Stevens et al., 2007); and robot design for remote, difficult-to-access areas such

13 as the deep sea and outer space, in which limited energy resources must be strictly conserved, are

14 areas of potential application.

15 MVT and the implementations described herein advance our understanding of how

16 animals should optimize sampling of their environment (Dall et al., 2005). The simulation model

17 we developed emphasizes the collection and use of personal information to reduce environmental

18 uncertainty; however, public information on habitat quality can also influence patch selection by

19 animals (Danchin et al., 1998; Danchin et al., 2004; Doligez et al., 2003; Doligez et al., 2004;

20 King \& Colishaw, 2007), including waterfowl (Pöysä, 2003; Ringelman et al., 2016). Moreover,

21 the degree to which animals learn about their environment and continually adjust their

22 assessments (Bayesian updating) remains an important topic in animal behavior (Oaten, 1977;

23 Green, 1980; Koops \& Abrahams, 2003; Valone, 2006; Trimmer et al., 2011). Our model of 
1 foraging waterfowl could be extended to model birds flocking (sensu Thorn, 2003) to a

2 previously profitable foraging patch (public information and group foraging) or by dynamically

3 adjusting the softmax temperature in response to a changing environment (Bayesian updating).

4 In this simulation, we have shown that a reinforcement-learning paradigm that accounts

5 for patch-switching costs is more effective than several alternatives and approaches a theoretical

6 upper bound of effectiveness. This strongly suggests that foraging strategies must use adaptive

7 learning. While the empirical question of the strategies that are actually used by foraging animals

8 in patchy environments cannot be answered by simulations alone, simulations can be used to

9 evaluate the relative effectiveness of hypothesized strategies. In conservation applications, it will

10 ultimately be important to understand and predict outcomes based on actual foraging strategies.

11 Our approach allows the investigation and comparison of strategies such as MVT, social learning,

12 and decisions based on interactions with other foragers. In short, our models provide explicit

13 predictions about the pattern of patch depletion and relative foraging efficiency. Future empirical

14 research on forager patch utilization can draw on these results to investigate strategies of

15 adaptive foraging behavior and the interaction of those strategies with conservation management

16 scenarios. 


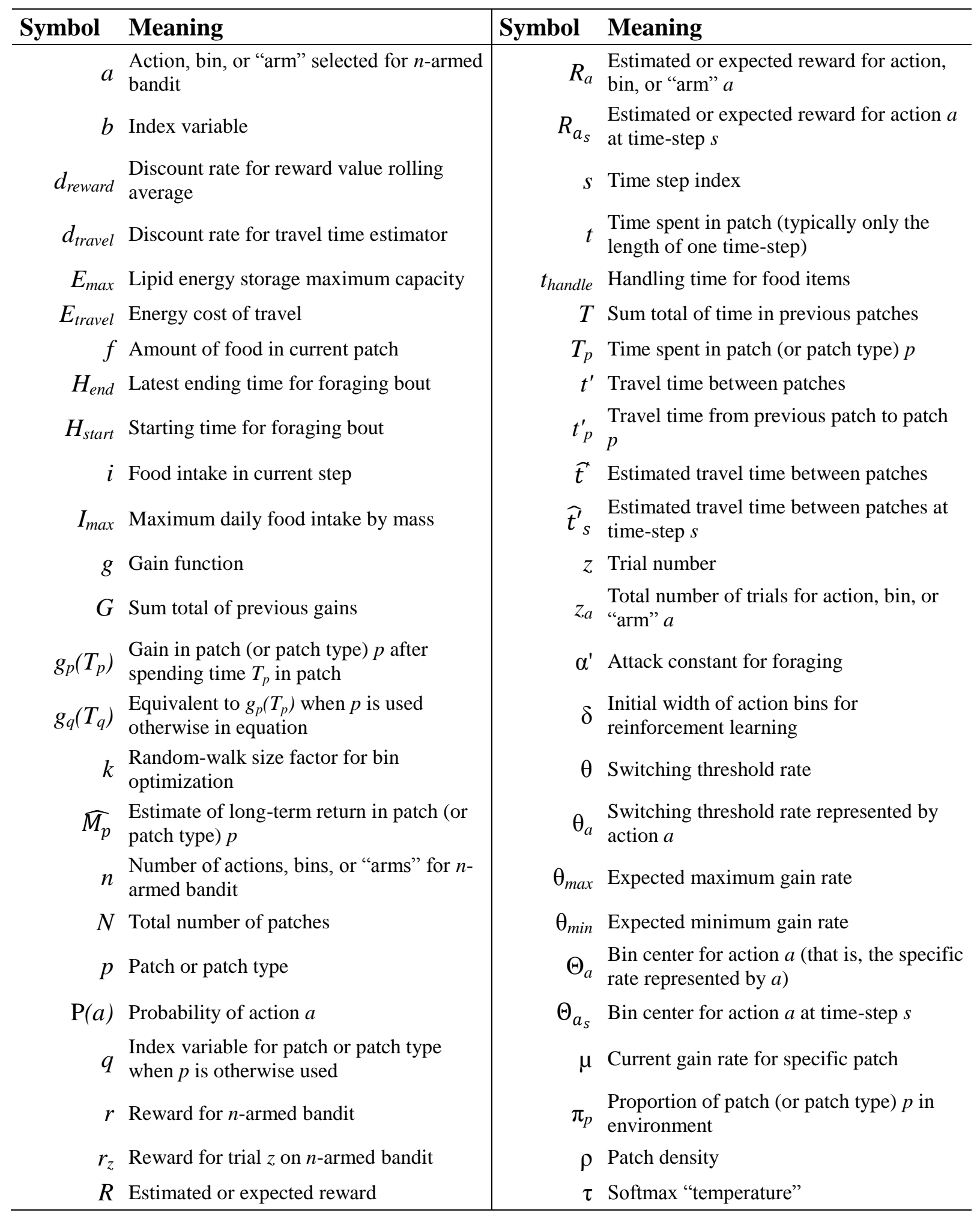

Table 5. Notation used in this report and its supplements. 
1 Acknowledgements

2 Author Contributions

3 M.M., J.E., and J.S. developed the concept for the research; M.M. and J.S. designed the research,

4 M.M. coded the model, M.M and K.R. analyzed the data, M.M., K.R., J.E., and J.S. wrote the

5 paper.

$6 \quad$ Funding

7 This work was supported by funds from Delta Waterfowl, the Dennis G. Raveling Endowed

8 Waterfowl Professorship, the University of California-Davis Provost's Fellowship, and the

9 National Science Foundation Graduate Fellowship grant \#DGE1148897.

\section{Conflict of interest}

11 The authors report no conflicts of interest. 


\section{References}

2 Albright, J.J., Owen, R.B., Corr, P.O., 1983. The effects of winter weather on the behavior and 3 energy reserves of black ducks in Maine. Trans. Northeast Sect. Wildl. Soc. 40, 118-128.

4 Bartoń, K.A., Hovestadt, T., 2013. Prey density, value, and spatial distribution affect the 5 efficiency of area-concentrated search. J. Theor. Biol. 316, 61-69.

6 Bernstein, C., Kacelnik, A., Krebs, J.R., 1988. Individual decisions and the distribution of 7 predators in a patchy environment. J. Anim. Ecol. 57, 1007-1026.

8 Bernstein, C., Kacelnik, A., Krebs, J.R., 1991. Individual decisions and the distribution of 9 predators in a patchy environment. II. The influence of travel costs and structure of the 10 environment. J. Anim. Ecol. 60, 205-225.

11 Central Valley Joint Venture, 2006. Central Valley Joint Venture Implementation Plan:

12 Conserving Bird Habitat. U.S. Fish and Wildlife Service.

13 Charnov, E.L., 1976. Optimal foraging, the marginal value theorem. Theor. Popul. Biol. 9, 129$14 \quad 136$.

15 Cowie, R.J., 1977. Optimal foraging in Great Tits. Nature 268, 137-139.

16 Cox, R.R., Afton, A.D., 1996. Evening flights of female northern pintails from a major roost site.

17 Condor 98, 810-819.

18 Dall, S.R., Giraldeau, L.-A., Olsson, O., McNamara, J.M., Stephens, D.W., 2005. Information

19 and its use by animals in evolutionary ecology. Trends Ecol. Evol. 20, 187-193.

20 Danchin, E., Boulinier, T., Massot, M., 1998. Conspecific reproductive success and breeding

21 habitat selection: Implications for the study of coloniality. Ecology 79, 2415-2428.

22 Danchin, E., Giraldeau, L.A., Valone, T.J., Wagner, R.H., 2004. Public information: From nosy 23 neighbors to cultural evolution. Science 305, 487-491.

24 DeAngelis, D., Mooij, W., 2005. Individual-based modeling of ecological and evolutionary 
processes. Annu. Rev. Ecol. Evol. S. 36, 147-168.

2 de Leeuw, J.J., 1996. Diving costs as a component of daily energy budgets of aquatic birds and

3 mammals: Generalizing the inclusion of dive-recovery costs demonstrated in tufted ducks.

4 Can. J. Zool. 74, 2131-2142.

5 Doligez, B., Cadet, C., Danchin, E., Boulinier, T., 2003. When to use public information for

6 breeding habitat selection? The role of environmental predictability and density dependence.

$7 \quad$ Anim. Behav. 66, 873-988.

8 Doligez, B., Part, T., Danchin, E. Clobert, T., Gustafsson, L., 2004. Availability and use of

9 public information and conspecific density for settlement decisions in the collared flycatcher.

$10 \quad$ J. Anim. Ecol. 73, 75-87.

11 Dooley, J.L., Sanders, T.A., Doherty, P.F. Jr., 2010. Mallard response to experimental walk-in 12 and shooting disturbance. J. Wildlife Manage. 74, 1815-1824.

13 Eadie, J.M., Elphick, C.S., Reinecke, K.J., Miller, M.R., 2008. Wildlife values of North

14 American ricelands. In: Manley, S.W. (Ed.), Conservation in Ricelands of North America. 15 The Rice Foundation.

16 Fleskes, J.P., Jarvis, R.L., Gilmer, D.S., 2002. Distribution and movements of female northern 17 pintails radiotagged in San Joaquin Valley, California. J. Wildlife Manage. 66, 138.

18 Fretwell, S.D., 1972. Populations in Seasonal Environments. Princeton University Press.

19 Fretwell, S.D., Lucas, H.L., 1969. On territorial behavior and other factors influencing habitat

20 distribution in birds. I. Theoretical development. Acta Biotheor. 19, 16-36.

21 Gibb, J.A., 1958. Predation by tits and squirrels on the eucosmid Ernarmonia conicolana. Anim.

$22 \quad$ Ecol. 27, 375-396.

23 Green, R.F., 1980. Bayesian birds: A simple example of Oaten’s stochastic model of optimal 
1 foraging theory. Theor. Popul. Biol. 18, 244-256.

2 Green, R.F., 1984. Stopping rules for optimal foragers. Am. Nat. 123, 30-43.

3 Grimm, V., Berger, U., Bastiansen, F., Eliassen, S., Ginot, V., Giske, J., Goss-Custard, J., Grand,

4 T., Heinz, S.K., Huse, G., Huth, A., Jepsen, J.U., Jørgensen, C., Mooij, W.M., Müller, B.,

5 Pe'er, G., Piou, C., Railsback, S.F., Robbins, A.M., Robbins, M.M., Rossmanith, E., Rüger,

6 N., Strand, E., Souissi, S., Stillman, R.A., Vabø, R., Visser, U., DeAngelis, D.L., 2006. A

7 standard protocol for describing individual-based and agent-based models. Ecol, Mod. 198,

$8 \quad 115-126$.

9 Grimm, V., Berger, U., DeAngelis, D.L., Polhill, J.G., Giske, J., Railsback, S.F., 2010. The ODD

10 protocol: A review and first update. Ecol. Mod. 221, 2760-2768.

11 Grimm, V., Revilla, E., Berger, U., Jeltsch, F., Mooij, W.M., Railsback, S.F., Thulke, H.,

12 Weiner, J., Wiegand, T., DeAngelis, D.L., 2005. Pattern-oriented modeling of agent-based

13 complex systems: Lessons from ecology. Science 310, 987-991.

14 Hodges, C.M., 1981. Optimal foraging in bumblebees: Hunting by expectation. Anim. Behav.

$15 \quad 29,1166-1171$.

16 Holling, C.S., 1959. Some characteristics of simple types of predation and parasitism. Can.

$17 \quad$ Entomol. 91, 385-398.

18 Iwasa, Y., Higashi, M., Yamamura, N., 1981. Prey distribution as a factor determining the choice

19 of optimal foraging strategy. Am. Nat. 117, 710-723.

20 King, A.J., Cowlishaw, G., 2007. When to use social information: The advantage of large group

21 size in individual decision making. Biol. Lett. 3, 137-139.

22 King, J.R., 1974. Seasonal allocation of time and energy resources in birds. In: Painter, R.A. Jr.

23 (Ed.), Avian Energetics. Nuttall Ornithological Club. 
1 Koops, M.A., Abrahams, M.V., 2003. Integrating the roles of information and competitive

2 ability on the spatial distribution of foragers. Am. Nat 161, 586-600.

3 Krebs, J.R., 1973. Behavioral aspects of predation. In: Bateson, P.P.G., Klopfer, P.H. (Eds.),

$4 \quad$ Perspectives in Ethology. Plenum Press.

5 Krebs, J.R., Ryan, J., Charnov, E.L., 1974. Hunting by expectation or optimal foraging: A study

6 of patch use by chickadees. Anim. Behav. 22, 953-964.

7 Lancaster, J.D., Davis, J.B., Kaminski, R.M., Afton, A.D., Penny, E.J., 2015. Mallard use of

8 managed public hunting area in Mississippi. J. Southeast. Assoc. Fish Wildl. Agencies 2,

$9281-287$.

10 Loesch, C.R., Kaminski, R.M., 1989. Winter body-weight patterns of female Mallards fed

11 agricultural seeds. J. Wildlife Manage. 53, 1081-1087.

12 Luke, S., Cioffi-Revilla, C., Panait, L., Sullivan, K., Balan, G., 2005. MASON: A multiagent

13 simulation environment. Simulation 81, 517-527.

14 McLane, A.J., Semeniuk, C., McDermid, G.J., Marceau, D.J., 2011. The role of agent-based

15 models in wildlife ecology and management. Ecol. Mod. 222, 1544-1556.

16 McNair, J.N., 1982. Optimal giving-up times and the marginal value theorem. Am. Nat. 119, $17 \quad 511-529$.

18 McNamara, J.M., 1982. Optimal patch use in a stochastic environment. Theor. Popul. Biol. 21, $19 \quad 269-288$.

20 McNamara, J.M., Houston, A.I., 1985. Optimal foraging and learning. J. Theor. Biol. 117, 231 21249.

22 Miller, M.L., Ringelman, K.M., Schank, J.C., Eadie, J.M., 2013. SWAMP: An agent-based 23 model for wetland and waterfowl conservation management. Simulation 90, 52-68. 
1 Miller, M.R., Eadie, J.M., 2006. The allometric relationship between resting metabolic rate and

2 body mass in wild waterfowl (Anatidae) and an application to estimation of winter habitat

3 requirements. Condor 108, 166-177.

4 Miller, M.R., Newton, W.E., 1999. Population energetics of northern pintails wintering in the

5 Sacramento Valley, California. J. Wildlife Manage. 63, 1222-1238.

6 Naylor, L.W., Master's Thesis 1999. Evaluating moist-soil seed production and management in

7 Central Valley wetlands to determine habitat needs for waterfowl. University of California,

8 Davis, CA.

9 Nolet, B.A., Gyimesi, A., Klaassen, R.H.G., 2006. Prediction of bird-carrying capacity on a

10 staging site: A test of depletion models. J. Anim. Ecol. 75, 1285-1292.

11 Oaten, A., 1977. Optimal foraging in patches: A case for stochasticity. Theor. Popul. Biol. 12, $12 \quad 263-285$.

13 Pierce, G.J., Ollason, J.G., 1987. Eight reasons why optimal foraging theory is a complete waste 14 of time. Oikos 49, 111-117.

15 Pöysä, H., 2003. Parasitic common goldeneye (Bucephala clangula) females lay preferentially in 16 safe neighbourhoods. Behav. Ecol. Sociobiol. 54, 30-35.

17 Prange, H.D., Schmidt-Nielsen, K., 1970. The metabolic cost of swimming in ducks. J. Exp.

18 Biol. 53, 763-777.

19 R Core Team, Software 2013. R: A language and environment for statistical computing (version

20 3.0.2). Vienna, Austria: R Foundation for Statistical Computing. Available from

21 http://www.R-project.org

22 Rave, D.P., Cordes, C.L., 1993. Time-activity budget of northern pintails using nonhunted rice

23 fields in southwest Louisiana. J. Field Ornithol. 64, 211-218. 
1 Ringelman, K.M., Eadie, J.M., Ackerman, J.T., Sih, A., Loughman, D.L., Yarris, G.S.,

2 Oldenburger, S.L, McLandress, M.R., 2016. Spatiotemporal patterns of duck nest density and

3 predation risk: A multi-scale analysis of 18 years and more than 10000 nests. Oikos.

$4 \quad$ doi:10.1111/oik.03728

5 Schmidt-Nielsen, K., 1972. Locomotion: Energy cost of swimming, flying, and running. Science $6 \quad 177,222-228$.

7 Sih, A., 1984. The behavioral response race between predator and prey. Am. Nat. 123, 143-150.

8 Sih, A., 1998. Game theory and predator-prey response races. In: Dugatkin, L.A., Reeve, H.K.

9 (Eds.), Game Theory and Animal Behavior. Oxford University Press.

10 Stephens, D.W., Brown, J.S., Ydenburg, R.C. (Eds.), 2007. Foraging: Behavior and Ecology.

11 University of Chicago Press.

12 Stephens, D.W., Krebs, J.R., 1987. Foraging Theory. Princeton University Press.

13 Stillman, R.A., Goss-Custard, J.D., Clarke, R.T., Durell, S.E.A. le V. dit, 1996. Shape of the

14 interference function in a foraging vertebrate. J. Anim. Ecol. 65, 813-824.

15 Sullivan, M.S., 1994. Mate choice as an information gathering process under time constraint:

16 Implications for behaviour and signal design. Anim. Behav. 47, 141-151.

17 Sutherland, W.J., 1996. From Individual Behaviour to Population Ecology. Oxford University 18 Press.

19 Sutton, R.S., Barto, A.G., 1998. Reinforcement Learning: An Introduction. MIT Press.

20 Thomas, D.R., Master's Thesis 2004. Assessment of waterfowl body condition to evaluate the 21 effectiveness of the Central Valley Joint Venture. University of California, Davis, CA.

22 Thorn, A., Master's Thesis 2003. Considerations in spatially explicit, individual based modeling 23 of waterfowl foraging behaviors. University of California, Davis, CA. 
1 Trimmer, P.C., Houston, A.I., Marshall, J.A.R., Mendl, M.T., Paul, E.S., McNamara, J.M., 2011.

2 Decision-making under uncertainty: Biases and Bayesians. Anim. Cogn. 14, 465-476.

3 Ulam, P., Balch, T., 2004. Using optimal foraging models to evaluate learned robotic foraging

4 behavior. Adapt. Behav. 12, 213-222.

5 Valone, T.J., 2006. Are animals capable of Bayesian updating? An empirical review. Oikos 112, $6 \quad 252-259$.

7 Wajnberg, E., Bernhard, P., Hamelin, F., Boivin, G., 2006. Optimal patch time allocation for 8 time-limited foragers. Behav. Ecol. Sociobiol. 60, 1-10.

9 Wawerla, J., Vaughan, R.T., 2009. Robot task switching under diminishing returns. In: IROS 10 2009: Proceedings of the IEEE/RSJ International Conference on Intelligent Robots and 11 Systems. IEEE.

12 Wawerla, J., Vaughan, R.T., 2010. Online robot task switching under diminishing returns. In:

13 Artificial Life XII: Proceedings of the Twelfth International Conference on the Synthesis and 14 Simulation of Living Systems. MIT Press.

15 Wooley, J.B., Owen, R.B., 1978. Energy costs of activity and daily energy expenditure in the 16 black duck. J. Wildlife Manage. 42, 739. 


\section{Supplementary Material}

\section{Appendix A}

3 A complete description of the underlying agent-based model ( $\mathrm{ABM}$ ) is provided by

4 Miller and colleagues (2013), from which this is an adaptation; included therein is a systematic

5 description following the ODD protocol (Overview, Design, Details; Grimm et al., 2006, 2010).

6 Here, we provide a brief overview of the model, including elements modified for the research

7 described in the main text.

8 The ABM was implemented in Java and MASON (Luke et al., 2005), a library for the

9 development of spatially-explicit, agent-based models. We developed code in the Eclipse

10 integrated development environment and compiled it in Oracle's Java Standard Edition 7

11 compiler (version 1.7.0_03) as a runnable JAR file. We ran the simulation experiments described

12 herein on a modeling farm of 12 identically-configured Windows 7 machines with Intel i5

13 processors and 2GB of RAM. Model code was executed in Oracle's Java Runtime Environment

14 SE 7 (version 1.7.0_03-b05).

$15 \quad$ A.1 Initialization 
When the model started, the landscape was initialized as a $10 \mathrm{~km}$ by $10 \mathrm{~km}$ square. A 2

$2 \mathrm{~km}$ by $2 \mathrm{~km}$ patch at the center was defined as the home refuge, the location at which agents

3 spent time while not foraging. The remainder of the landscape was divided into $1 \mathrm{~km}$ by $1 \mathrm{~km}$

4 foraging or non-foraging patches arranged in order from top to bottom then from left to right in a

5 regular pattern. Each patch was specified to be foraging or non-foraging so that the proportion of

6 forageable patches matched the proportion specified in the setup parameters as closely as

Figure A 1 Visualization of the ABM at runtime. Agents are represented as dots, the home refuge is shown with horizontal hatching, rice fields are shown with diagonal hatching, and moist soil patches are shown with vertical hatching. At initialization (a), the refuge appears solid black because it is covered with agents. As agents move out to foraging locations (b), groups appear as multiple agents travel to the same patch. In (c), foragers are located on target patches during foraging.

7 possible. Foraging patches contained food and were possible targets for agents during foraging;

8 non-foraging patches had no food and were never targets for agents. Foraging patches could be

9 either rice patches or moist-soil patches; this determined what kind of food was present in the

10 patch and the density of that food. Patches selected as foraging habitats were assigned as follows:

11 the new foraging patch would be a rice patch until the number of rice patches exceeded the

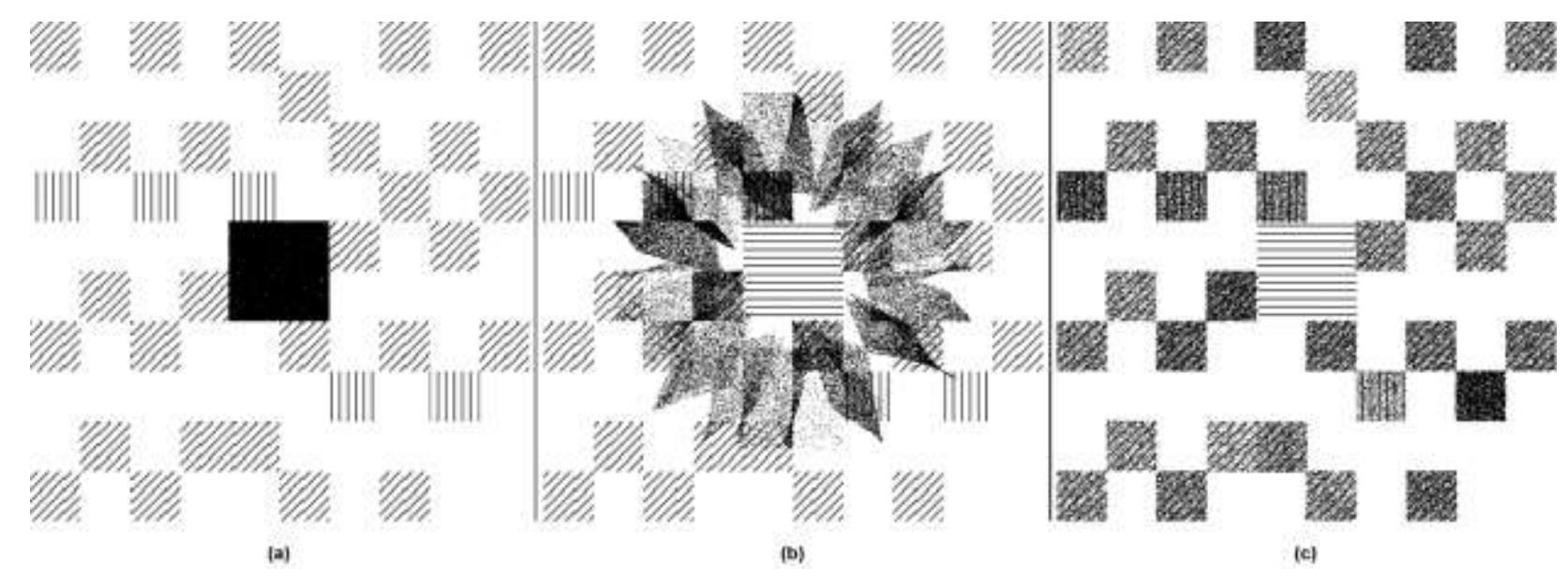

(a)

(b)

(c)

12 specified rice proportion, at which point the new patch would be a moist-soil patch. Figure A1(a)

13 shows a representative landscape setup with forageable proportion at 0.35 and rice fields at 0.86

14 of the forageable landscape. Initial landscape values were based on reported field observations

15 (table A1); food items were also modeled based on reported values (table A2). Note in table A2 
1 that moist soil seed aggregate refers to a combination of the primary seeds found in moist soils in

2 California's Central Valley represented as a weighted average. Some parameters varied for

3 different experiments; the specific values used are discussed in the main text.

\begin{tabular}{llll}
\hline Parameter & Initial Value & Units & Source \\
\hline $\begin{array}{l}\text { Forageable proportion } \\
\begin{array}{l}\text { Moist soil as proportion of } \\
\text { forageable landscape }\end{array}\end{array}$ & Varied by experiment & Varied by experiment & (see main text) \\
$\begin{array}{l}\text { Moist soil patch food } \\
\text { density }\end{array}$ & $\begin{array}{l}\text { Drawn from normal distribution, } \\
\mu=58.5, \sigma=6.6\end{array}$ & $\mathrm{~g} / \mathrm{m}^{2}$ & Naylor, 1999 \\
$\begin{array}{l}\text { Rice patch food density } \\
\text { Drawn from normal distribution, } \\
\mu=33.3, \sigma=2.2\end{array}$ & $\mathrm{~g} / \mathrm{m}^{2}$ & Eadie et al., 2008 \\
\hline
\end{tabular}

Table A 1 Initial Patch Parameters

\begin{tabular}{lll}
\hline Parameter & Moist-soil seed aggregate & Rice \\
\hline True metabolizable energy* $(\mathrm{kcal} / \mathrm{g})$ & 3.4 & 3.53 \\
Attack constant $\left(\mathrm{m}^{2} / \mathrm{s}\right), \alpha^{\prime}$ & $2 \times 10^{-4}$ & $2 \times 10^{-4}$ \\
Handling time** $(\mathrm{s})$, thandle & 2.0 & 2.0 \\
\hline
\end{tabular}

Table A 2 Initial food type parameters. *Eadie et al., 2008. **Sutherland, 1996; Thorn, 2003.

5 At simulation start, 80,000 agents were placed in the refuge at random, maintaining

6 sufficient distance to prevent exceeding one agent per square meter. Agents started with lipid

7 energy store drawn from a normal distribution; mean and variance were based on empirical data

8 (Thomas, 2004). Agents began each simulation with values for food eaten that day and non-lipid

9 energy reserve set to 0 . The initial state of the agents was based on reported values or were

10 varied by experiment; some values not available in the literature were based on field observation

11 (table A3). 


\begin{tabular}{|c|c|c|c|c|}
\hline Parameter & Initial Value & Units & Symbol* & Source \\
\hline Resting metabolic rate & 0.0010643017 & $\mathrm{kcal} / \mathrm{s}$ & & Miller \& Eadie, 2006 \\
\hline Flying metabolic rate & $12.0 \times$ resting rate & $\mathrm{kcal} / \mathrm{s}$ & & $\begin{array}{l}\text { Prange \& Schmidt- } \\
\text { Nielsen, 1970; Schmidt- } \\
\text { Nielsen, 1972; King, } \\
1974\end{array}$ \\
\hline Foraging metabolic rate & $2.5 \times$ resting rate & $\mathrm{kcal} / \mathrm{s}$ & & Miller et al., 2013 \\
\hline Non-foraging metabolic rate & $2.0 \times$ resting rate & $\mathrm{kcal} / \mathrm{s}$ & & Miller et al., 2013 \\
\hline Daily food intake capacity & 100.0 & $\mathrm{~g}$ & $I_{\max }$ & Miller et al., 2013 \\
\hline Maximum lipid energy store & 3148.25 & kcal & $E_{\max }$ & Thomas, 2004 \\
\hline $\begin{array}{l}\text { Maximum forager density in } \\
\text { refuge }\end{array}$ & 1.0 & $\mathrm{~m}^{-2}$ & & (field observations) \\
\hline $\begin{array}{l}\text { Maximum forager density in } \\
\text { foraging patches }\end{array}$ & 1.0 & $\mathrm{~m}^{-2}$ & & (field observations) \\
\hline Earliest daily foraging time & 18.0 & & $H_{\text {start }}$ & $\begin{array}{l}\text { Cox \& Afton, 1996; } \\
\text { Fleskes et al., } 2002\end{array}$ \\
\hline Latest daily foraging time & 6.0 & & $H_{\text {end }}$ & $\begin{array}{l}\text { Cox \& Afton, 1996; } \\
\text { Fleskes et al., } 2002\end{array}$ \\
\hline Giving-up density & 5.6 & $\mathrm{~g} / \mathrm{m}^{2}$ & & $\begin{array}{l}\text { Naylor, 1999; Central } \\
\text { Valley Joint Venture, } \\
2006\end{array}$ \\
\hline Lipid energy store & $\begin{array}{l}\text { Drawn from normal } \\
\text { distribution, } \\
\mu=1349.25, \sigma=179.9\end{array}$ & kcal & & Thomas, 2004 \\
\hline $\begin{array}{l}\text { Mass of food eaten during } \\
\text { current/most recent foraging } \\
\text { bout }\end{array}$ & 0.0 & $\mathrm{~g}$ & & (see text) \\
\hline $\begin{array}{l}\text { Food energy consumed during } \\
\text { current/most recent foraging } \\
\text { bout }\end{array}$ & 0.0 & kcal & & (see text) \\
\hline
\end{tabular}

Table A 3 Initial agent parameters. *Symbols are only provided for parameters mentioned in the text.

\section{$2 \quad$ A.2 Model Runtime}

$3 \quad$ Figure A2 and A3 are flowcharts summarizing overall model progress and individual

4 forager behavior; Figures A5 through A8 (below) have pseudo-code for MVT decisions. 


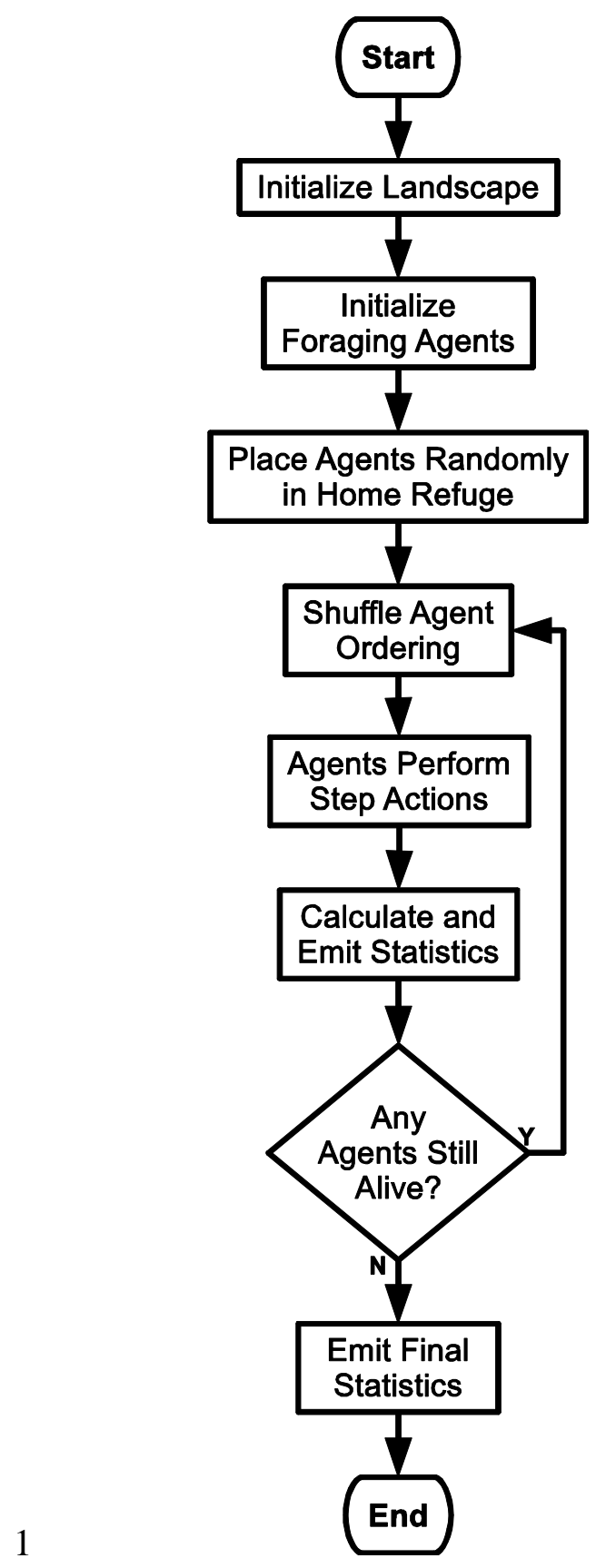

Figure A 2 Main Model Loop 


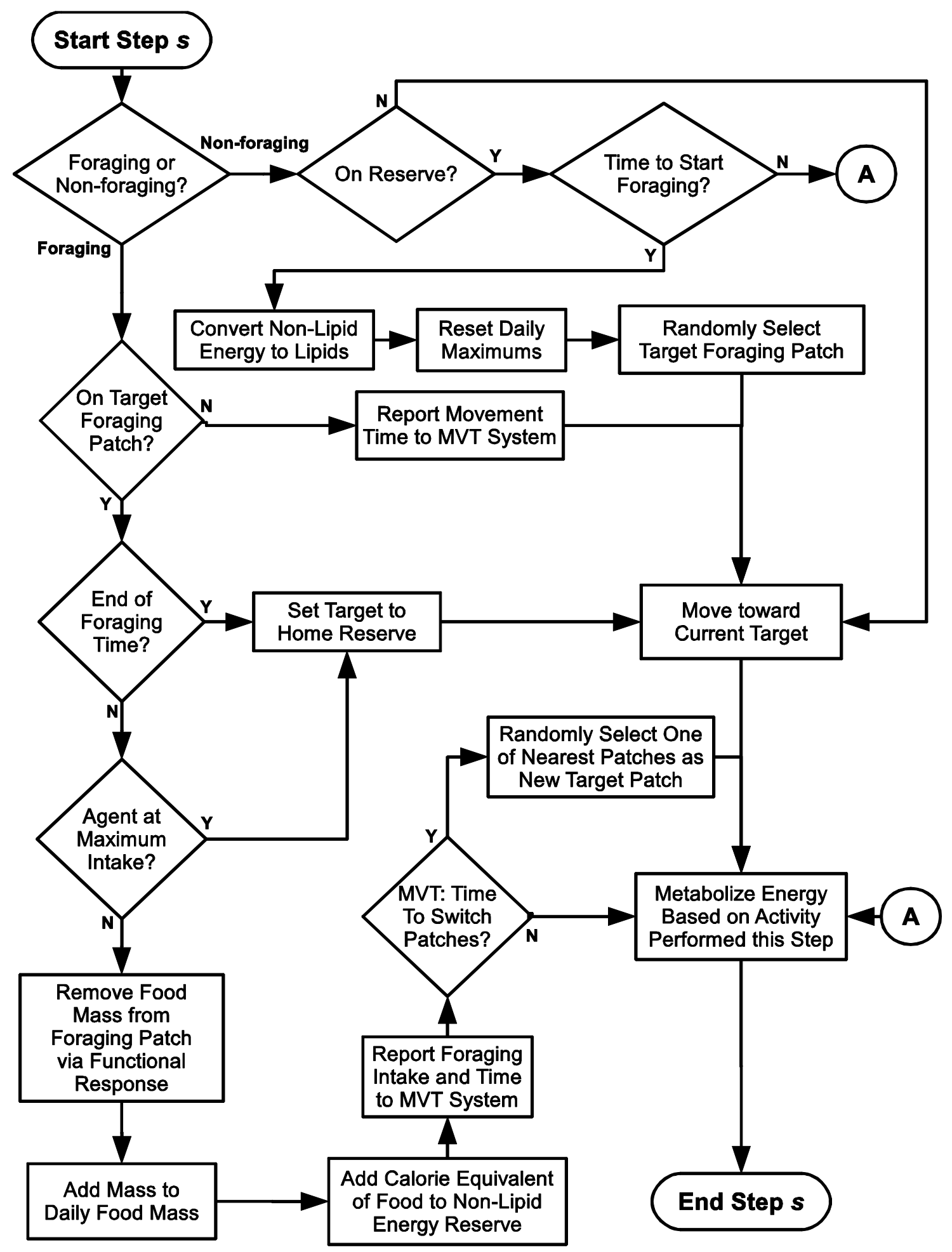

Figure A 3 Agent step flowchart

The ABM's basic agent was the forager. A forager agent had two top-level states:

2 foraging and roosting. While foraging, the agent simulated a hungry animal motivated to acquire 
1 food; while roosting, the agent simulated a satiated animal. In both states, the agent had a target

2 patch: during roosting, the home refuge, and while foraging, one of the forageable patches.

3 While the agent was not on its target patch, it moved in a straight line from its current location to

4 the nearest point of the target patch, modeling flight. When the agent was on its target patch, it

5 behaved as described below, depending on its top-level state. At each time step, an agent used

6 energy at the metabolic rate of its current behavior. Metabolic accounting details are described

7 below, following discussion of the foraging and roosting states.

\section{A.2.1 Foraging State}

An agent was in the foraging state while actively seeking and acquiring food to meet

overall metabolic demands. A foraging bout was a contiguous time period during which the agent

11 was in this state. Foraging bouts began when the time-of-day was between the foraging start time

$12\left(H_{\text {start }}\right)$ and the foraging end time $\left(H_{\text {end }}\right)$ provided that $H_{\text {end }}$ for the previous bout had passed. A

13 foraging bout could continue until $H_{\text {end }}$, but could also end earlier based upon foraging success if

14 either of the following were satisfied: (1) agents had a maximum daily food capacity $\left(I_{\max }\right)$ which

15 limited the food mass they could eat in one foraging bout; if the agent ate $I_{\max }$ or more at any

16 time step during a bout, the bout ended; (2) agents had a maximum lipid capacity ( $\left.E_{\max }\right)$ which

17 limited the amount of energy they could store as lipids; if the energy taken in during a bout met

18 or exceeded the energy required to bring the agent's lipid reserves to $E_{\max }$, the bout ended.

While the agent was actively foraging, it consumed food from its current foraging patch

20 at a rate dependent on the density of food in that patch. The ABM used Holling's (1959) disc

21 equation, a type II functional response:

$$
\frac{i}{t}=\frac{\alpha^{\prime} \rho}{1+\alpha^{\prime} \rho t_{\text {handle }}},
$$


1 in which $i$ was amount of food eaten, $t$ was elapsed time, $\alpha$ ' was attack constant, $\rho$ was the

2 density of food in the patch, and $t_{\text {handle }}$ was handling time for a unit of food $\left(H_{T}\right.$ in Holling, 1959$)$.

3 In this equation, attack constant was a measurement of search efficiency, expressed in area per

4 time $\left(\mathrm{m}^{2} / \mathrm{s}\right)$. Handling time (s) was the amount of time the agent need to manipulate a unit of

5 food after it was found. This resulted in agents acquiring food at a rate proportional to the density

6 of food, modified by search and handling efficiency. Equation A1 is rearranged for the purposes

7 of this model as

$$
i=\frac{\alpha^{\prime} \rho t}{1+\alpha^{\prime} \rho t_{\text {handle }}}
$$

9 Consumed food is removed immediately from the patch, thus decreasing the patch's density and

10 so lowering the foraging efficiency of agents subsequently foraging in the patch. The consumed

11 food is also added to the agent's account of mass eaten during the bout (to implement $I_{\max }$ ) and of

12 energy intake (to implement $E_{\max }$ and to provide non-lipid energy). Metabolic cost of foraging

13 behavior includes the cost of moving during foraging. If a patch's food density falls below the

14 simulation-wide, fixed giving up density during a foraging bout, the agent continues to utilize

15 that patch until the end of that bout or until the MVT algorithm indicates the agent should switch

16 patches (note, however, that such patches cannot be selected on subsequent bouts). Competition

17 between agents is indirect: depletion of resources by one agent reduces the efficiency of

18 subsequent agents on the same patch, since in Equation A2, $i$ decreases as $\rho$ decreases. Note that

19 this is effected within a time step: the decrease in patch density occurs immediately instead of at

20 the end of the time step, so that inter-agent exploitation competition occurs within that time step.

21 At the beginning of a foraging bout (described in the subsection on the roosting state,

22 below), each agent independently selects a foraging patch for the beginning of that bout. The 
1 selection for the upcoming bout is independent of previous selections. Two different methods are

2 used to select starting patch for each foraging bout, depending on the simulation. For MVT

3 experiments and for the control condition, agents were randomly distributed uniformly among

4 foraging patches. For experiments simulating a theoretical upper bound of optimal foraging via

5 ideal free distribution (IFD; Fretwell \& Lucas, 1969), the probability of selecting a patch is

6 proportional to the density of food on that patch at the beginning of the upcoming bout (Stillman

7 et al., 1996; Miller et al., 2013). Figure A1(c) shows foragers dispersed in foraging patches

8 during a foraging bout.

$9 \quad$ Agents in MVT experiments could change foraging patches within bouts (in IFD and

10 control experiments, agents did not change patches within a bout). The MVT algorithms received

11 information about ongoing foraging parameters (time spent traveling to patches, time spent in

12 patches, and amount of food obtained in patches) and returned a Boolean value indicating

13 whether or not the agent should switch patches at the next time step. If false was returned, the

14 agent remained in its current patch; if true was returned, the agent retrieved a list of the nearest

15 patches, selected one at random, and moved to that patch to continue foraging. This continued

16 until the normal end of the foraging bout, as described above. Four different algorithms were

17 available for MVT decisions: reinforcement learning (RLMVT), online (OMVT), extended

18 reinforcement learning (XRLMVT), and extended online (XOMVT). The main text describes the

19 logic and details of each of these methods of approximating MVT behavior; thus, the following

20 paragraphs provide implementation-specific details. Note that each agent collected and processed

21 data independently of the other agents and thus made decisions based only on individual

22 experience.

23 For RLMVT, the number of bins and thus the number of different switching rates $(n$ in 
1 main text Equations 5, 9, and 12) was set to 5; thus, agents using this algorithm had to switch

2 patches 5 times to initialize the bins before beginning to use the reinforcement learning

3 simulation in earnest. The softmax temperature $(\tau)$ was set to 15.0 , providing moderate but

4 modest exploration once an

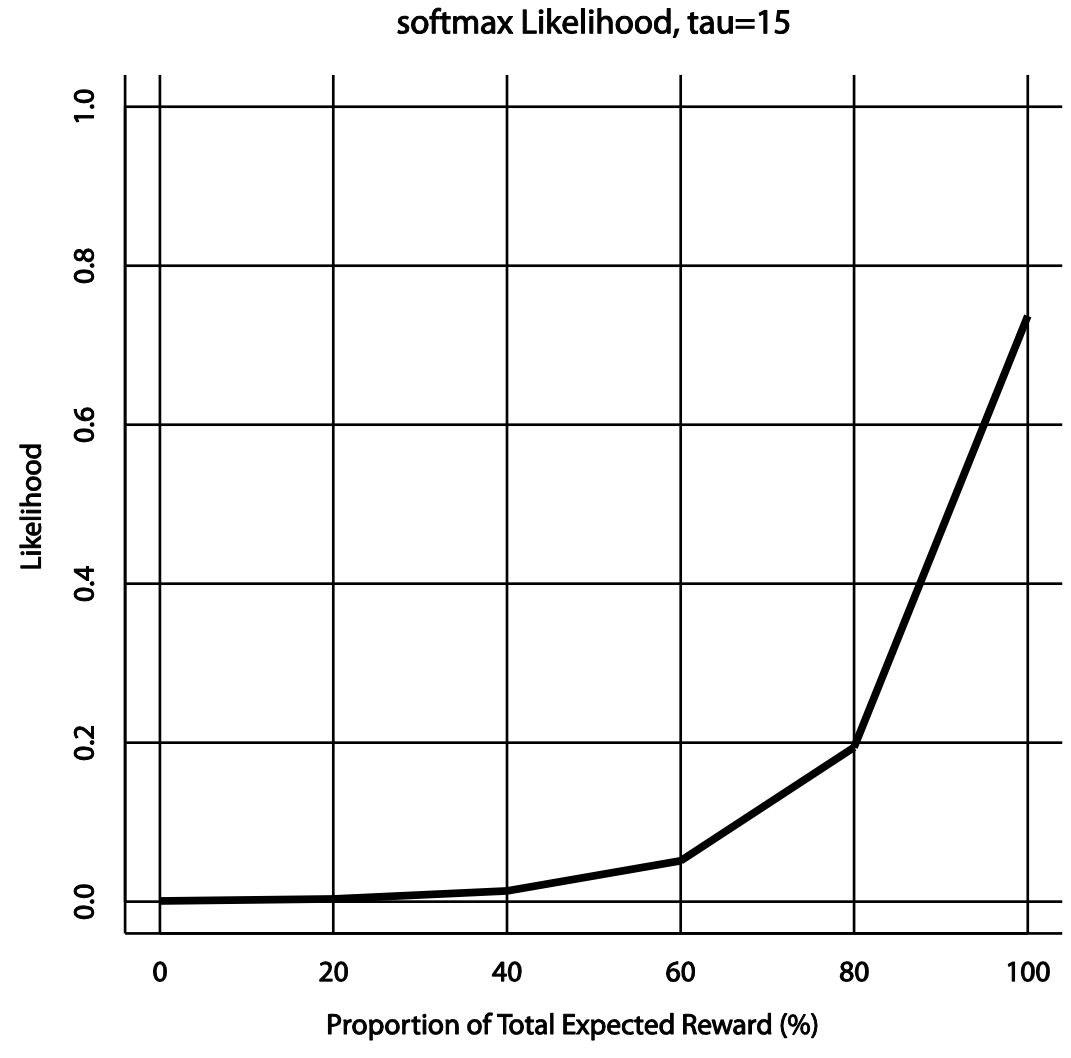

Figure A 4 Bin likelihood of selection by proportion of total expected reward with $\tau=15.0$. Note that sum total contributions to likelihood can exceed 1; thus, probability is scaled by the sum total of the contributions of all of the bins. optimal switching rate began

to emerge; Figure A4 is a

graph comparing a bin's

proportion of the total

combined expected reward

(that is, the bin's total reward

value divided by the

combined reward value of all

the bins) to that bin's portion

of the likelihood of being

selected by the softmax

algorithm.

The parameters used

to map the continuous space

19 of switching threshold onto the discrete mechanism of the $n$-armed bandit (see Appendix C. 1 for

20 rationale and details) included random-walk size factor $(k)$ and update factor for the expected

21 reward $\left(d_{\text {reward }}\right)$. The fixed value for $k$ was 0.25 , so the maximum exploration range away from

22 the current bin center for optimization was one quarter the initial bin width. The fixed value for

$23 d_{\text {reward }}$ was 0.5 , thus the most recent weight was averaged with the prior expected reward. Figure 
1 A5 provides pseudo-code

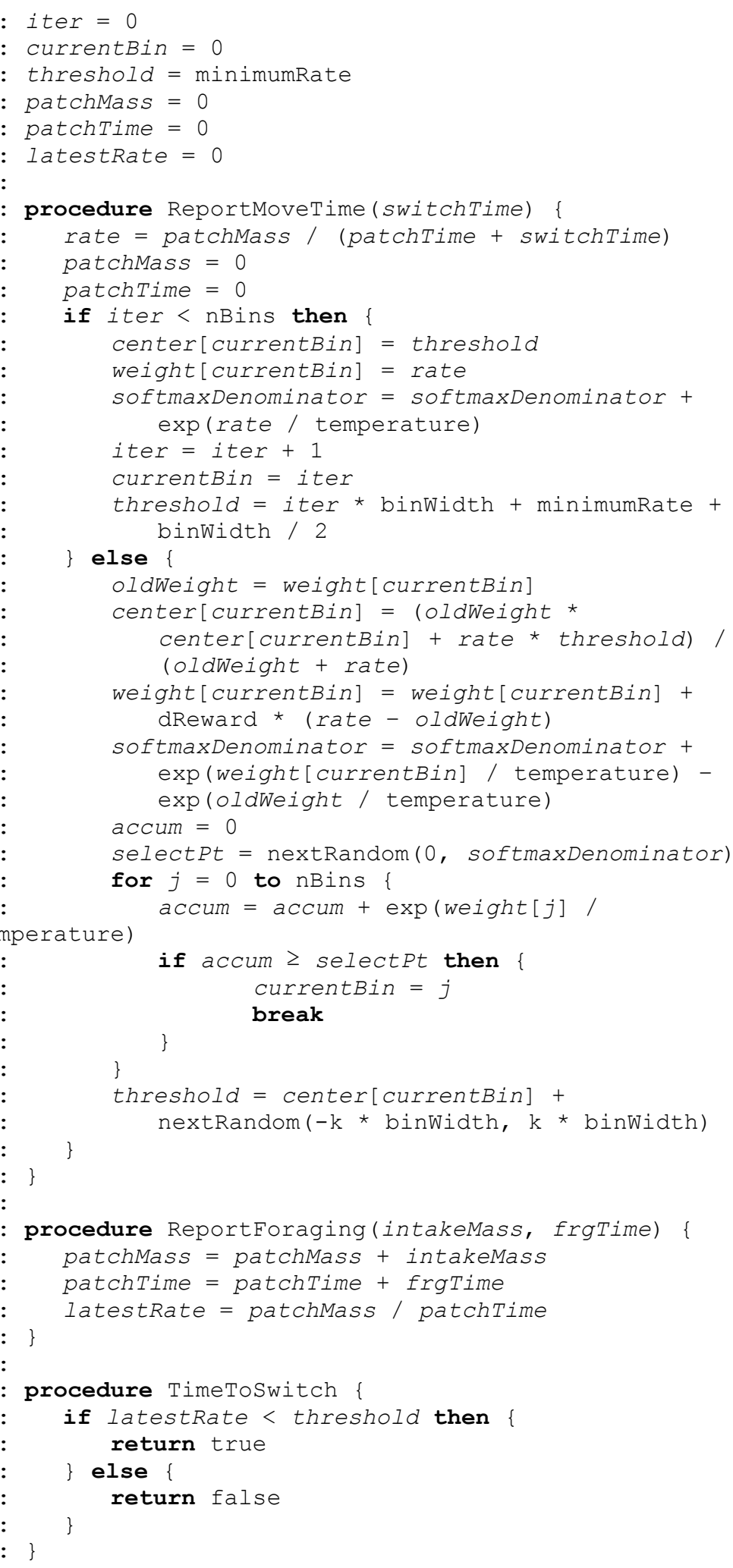

for RLMVT decision-

making.

For OMVT, the

only factor required was

the update factor for

estimated patch-switching

time $\left(d_{\text {travel }}\right)$. This was set

to 0.5 , so the most recent

switching time was

averaged with the prior

estimate. Figure A6

provides pseudo-code for

OMVT decision-making.

For XRLMVT, the

factors described above

were set to the same values

as described above for

RLMVT. Figure A7

provides pseudo-code for

XRLMVT decision-

making. Note the key

differences between the

Figure A 5 RLMVT algorithm pseudo-code 
1 RLMVT and the XRLMVT

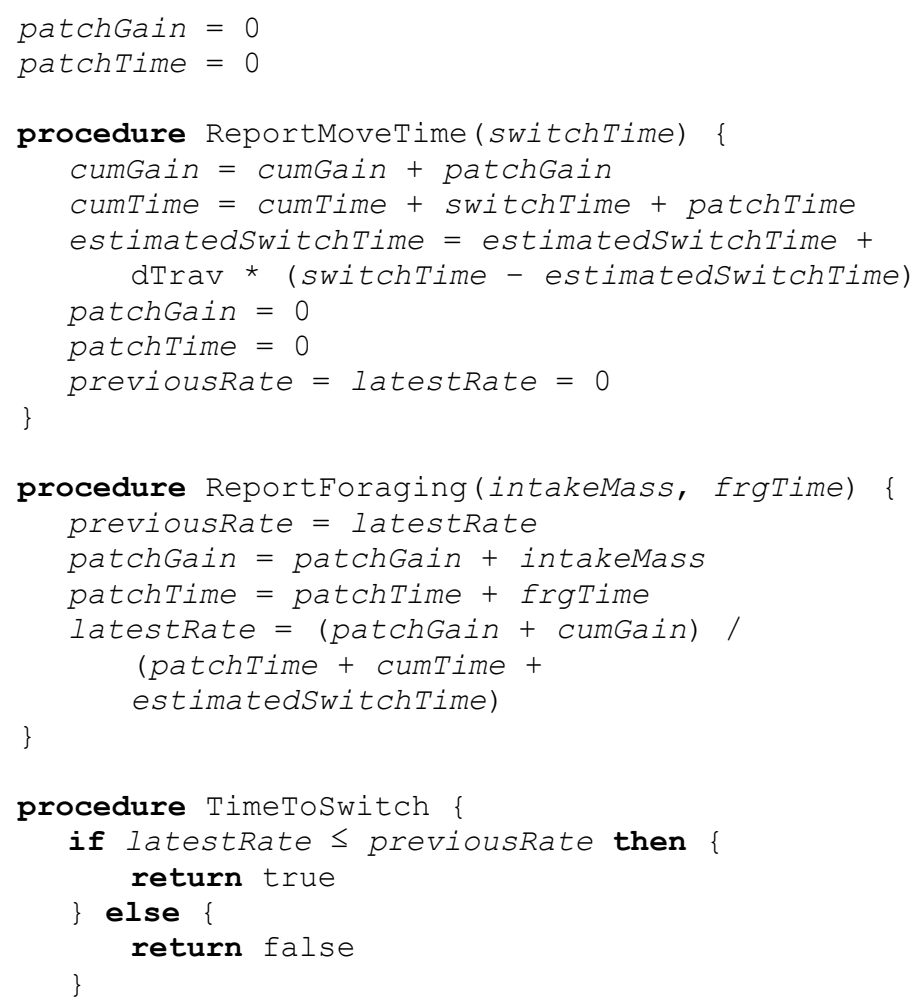

13 updating factor was set to the same value as for OMVT, as described above. Figure A8 provides

14 pseudo-code for XOMVT decision-making. The key differences between OMVT and XOMVT

15 appear in lines 9, 14, and 16, and are parallel to the differences between RLMVT and XRLMVT,

16 described above.

Figure A 6 OMVT algorithm pseudo-code

A.2.2 Roosting State 


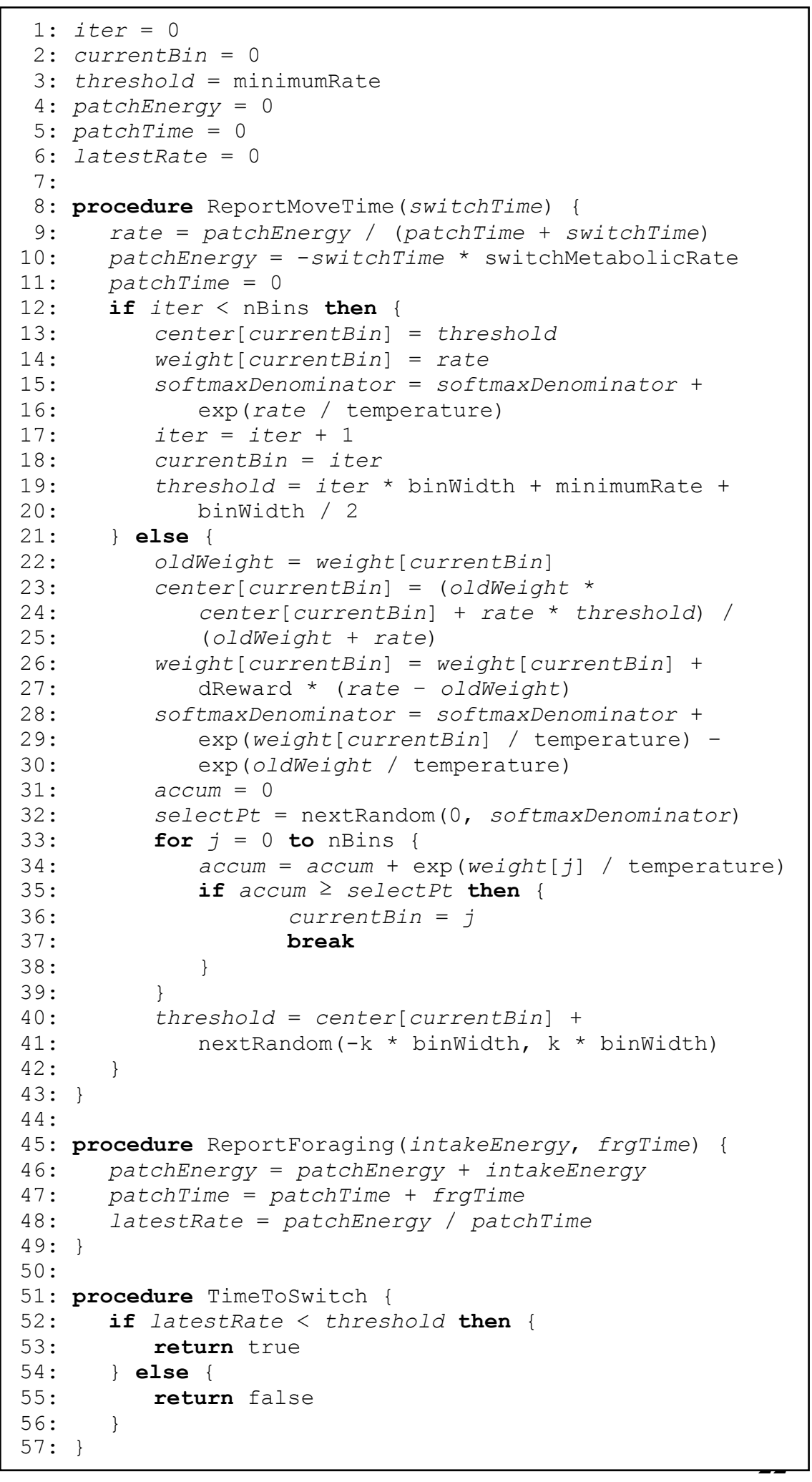

Figure A 7 XRLMVT algorithm pseudo-code
An agent was in the

roosting state while it was

not foraging or moving

from roosting areas to

foraging areas. This

behavioral state

encompassed resting, short

trips on water or land, short

flights, mating and

courtship, sleep, and other

behaviors. We modeled the

metabolic rate of the

roosting state as the

average metabolic rate of

these behaviors weighted

by the empirically-

observed time budget for

the behaviors (Table A4;

Rave \& Cordes, 1993). The

model included metabolic

costs of movement, but

individual agents did not

$\iota \quad$ change location after 
1 arriving at the refuge to increase simulation speed.

2 


\begin{tabular}{|c|c|c|c|c|}
\hline Behavior & $\begin{array}{l}\text { Proportion } \\
\text { time budget }\end{array}$ & $\begin{array}{l}\text { Multiplier } \\
\text { to RMR }\end{array}$ & Weighted & Multiplier source \\
\hline Sleep & 0.52 & 1.1 & 0.572 & Wooley \& Owen, 1978 \\
\hline Swimming and rest & 0.16 & 1.4 & 0.224 & Wooley \& Owen, 1978 \\
\hline Courtship & 0.06 & 2.4 & 0.144 & Albright, Owen, \& Corr, 1983 \\
\hline Flying & 0.05 & 12.5 & 0.625 & Wooley \& Owen, 1978 \\
\hline Diving & 0.00 & 5.1 & 0.000 & de Leeuw, 1996 \\
\hline Surfacing & 0.00 & 3.5 & 0.000 & Wooley \& Owen, 1978 \\
\hline Preening & 0.00 & 2.1 & 0.000 & Albright, Owen, \& Corr, 1983 \\
\hline Aggression & 0.00 & 1.8 & 0.000 & Wooley \& Owen, 1978 \\
\hline Sum & 0.79 & & 1.565 & \\
\hline Weighted Average & & & 1.981 & \\
\hline
\end{tabular}

Table A 4 Non-foraging metabolic rate components. Time budget proportions derived from Rave $\&$ Cordes, 1993; proportion does not sum to 1.0 because foraging behavior is included elsewhere.

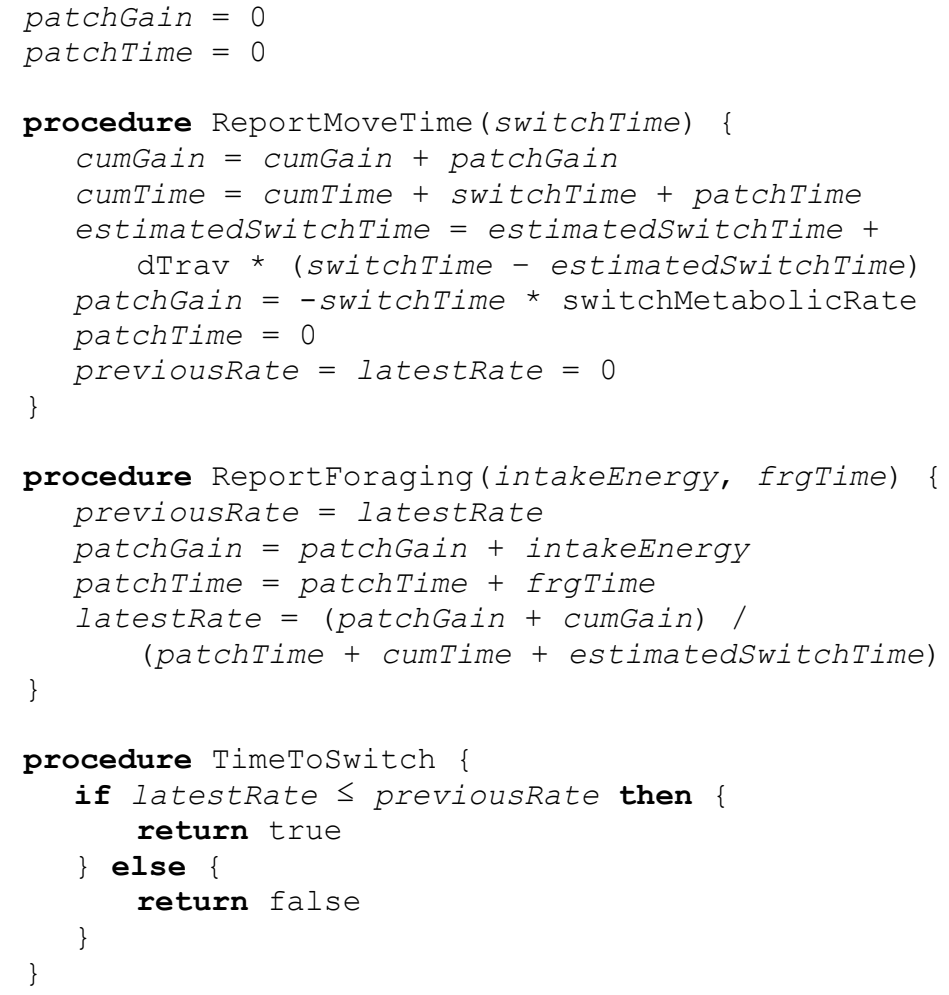

Figure A 8 XOMVT algorithm pseudo-code
The simulation began

with agents in the roosting state.

Roosting ended and foraging

began when the time of day was

later than $H_{\text {start }}$ given that $H_{\text {end }}$

for the previous foraging bout

had passed. When roosting

ended, each agent selected a

new foraging location

independently. The roosting

state began when foraging

13 ended, as described above. 


\section{A.2.3 Metabolism}

Agents had two separate energy reserves: non-lipid energy reserve and lipid stores. Nonlipid energy reserve modeled blood glucose: energy from consumed food accumulated in this reserve and this reserve was the primary supply for metabolic demand (note that this reserve did not impose a lipid-conversion cost). Lipid stores modeled the agent's lipid reserves, or body fat.

When an agent removed food from a foraging patch, the metabolizable energy of the food was immediately added to the non-lipid energy reserve. The non-lipid energy reserve was maintained through the current foraging bout and the subsequent roosting state, with the metabolic demand for that time subtracted. At the beginning of the next foraging bout, before the agent began flight to its target foraging patch, all remaining non-lipid energy reserves were moved to lipid stores, subtracting an amount that modeled the cost of the interconversion of glucose and lipids (blood glucose was converted to lipids at $16.747 \mathrm{kcal} / \mathrm{g}$; lipids were converted back to blood glucose at $8.995 \mathrm{~g} / \mathrm{kcal}$; this resulted in an interconversion efficiency of 0.537 ;

Miller \& Newton, 1999). The lipid store had a maximum capacity, $E_{\max }$ (Thomas, 2004). The non-lipid energy reserve satisfied metabolic demand first. If that reserve was

16 insufficient, lipid stores satisfied the remaining demand. When both of these reserves reached 0 $7 \mathrm{kcal}$, the agent died. When all agents died, the simulation ended. 


\section{Appendix B}

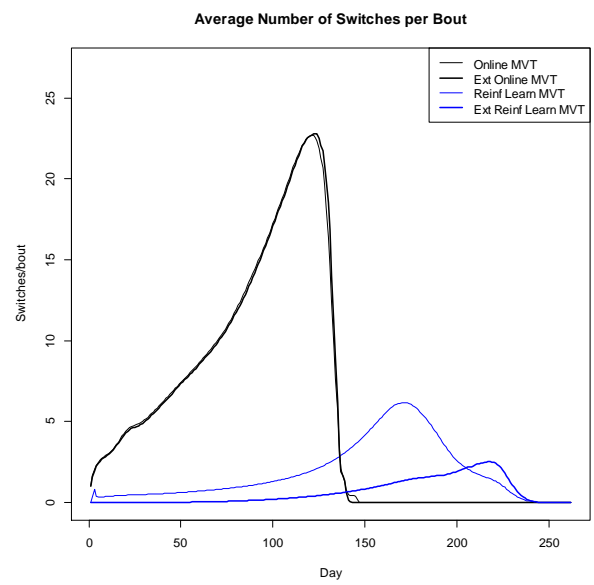

(a)

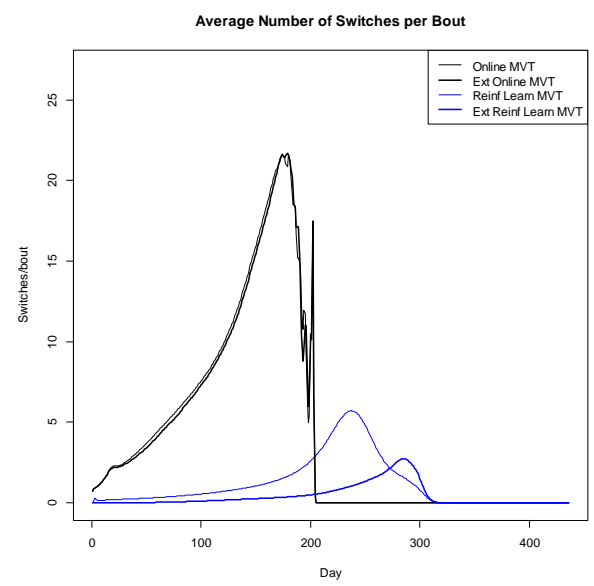

(c)

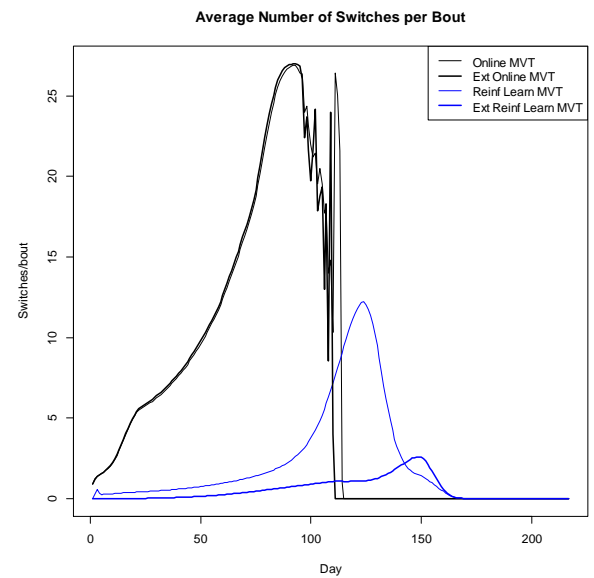

(e)

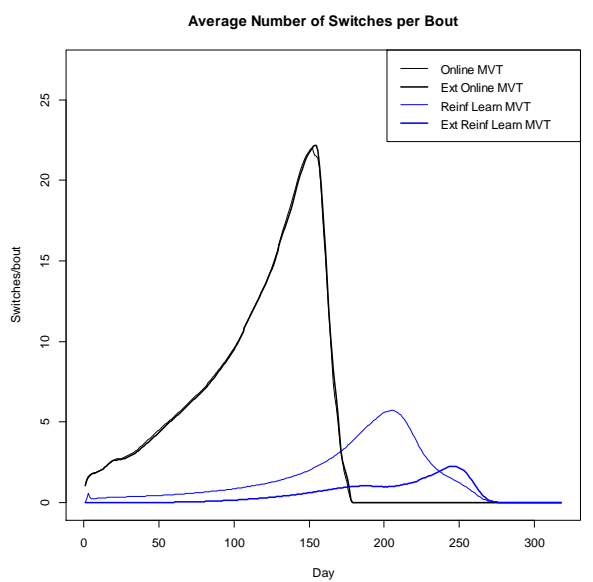

(b)

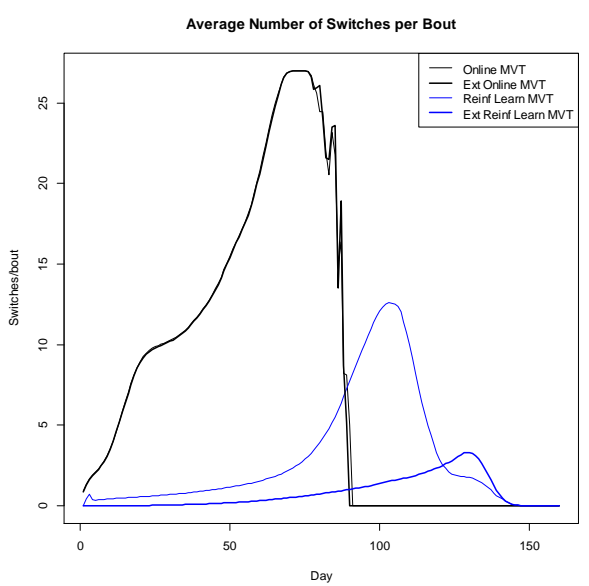

(d)

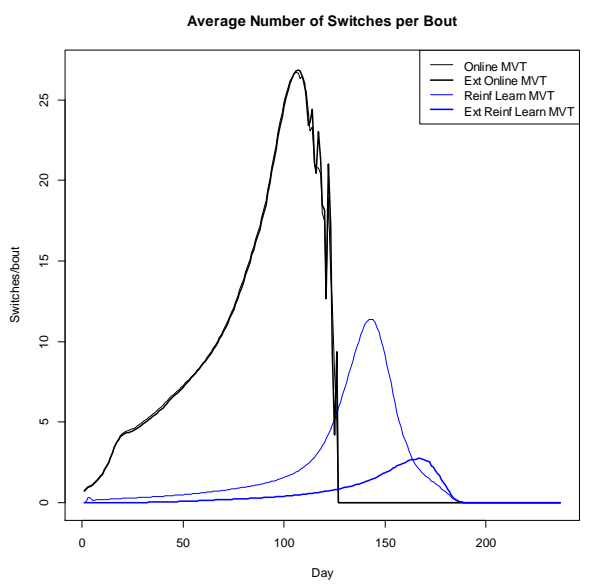

(f)

Figure B 1. Number of switches compared between MVT strategies for all landscape conditions: (a) 2700 ha rice, 700 ha moist soil; (b) 2000 ha rice, 1400 ha moist soil; (c) richest -1400 ha rice, 2000 ha moist soil; (d) poorest -1600 ha rice, 400 ha moist soil; (e) 1200 ha rice, 800 ha moist soil; and (f) 800 ha rice, 1200 ha moist soil. 


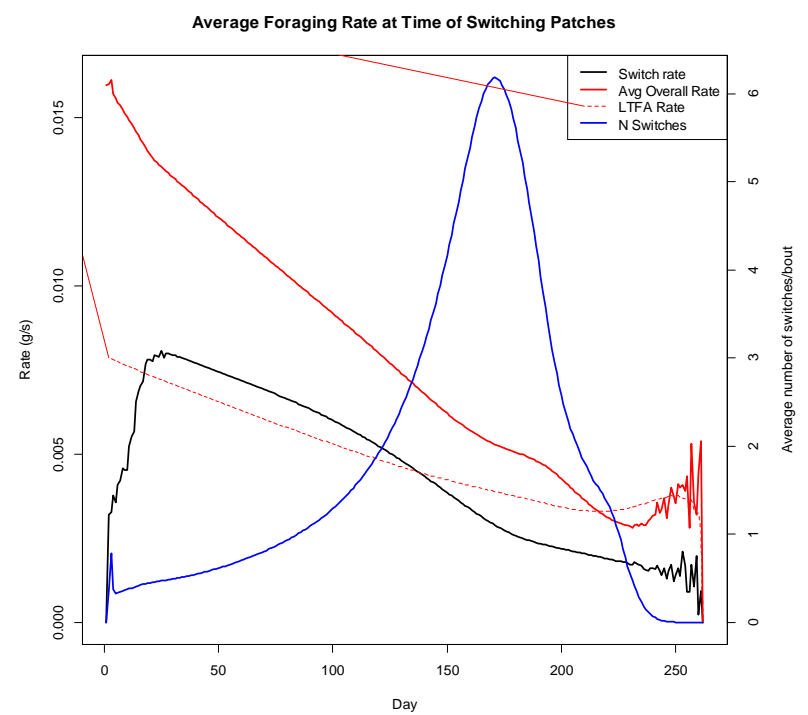

(a)

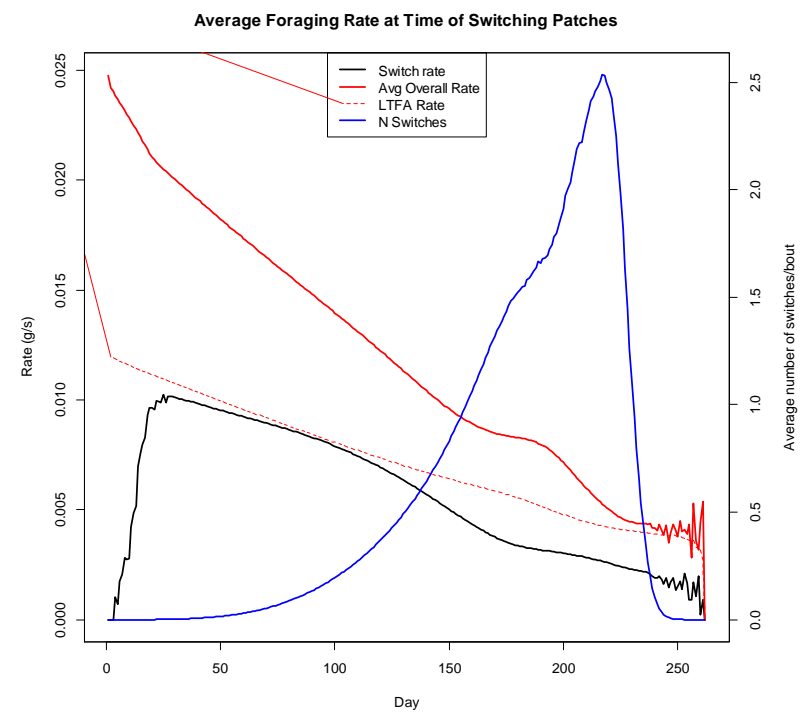

(c)

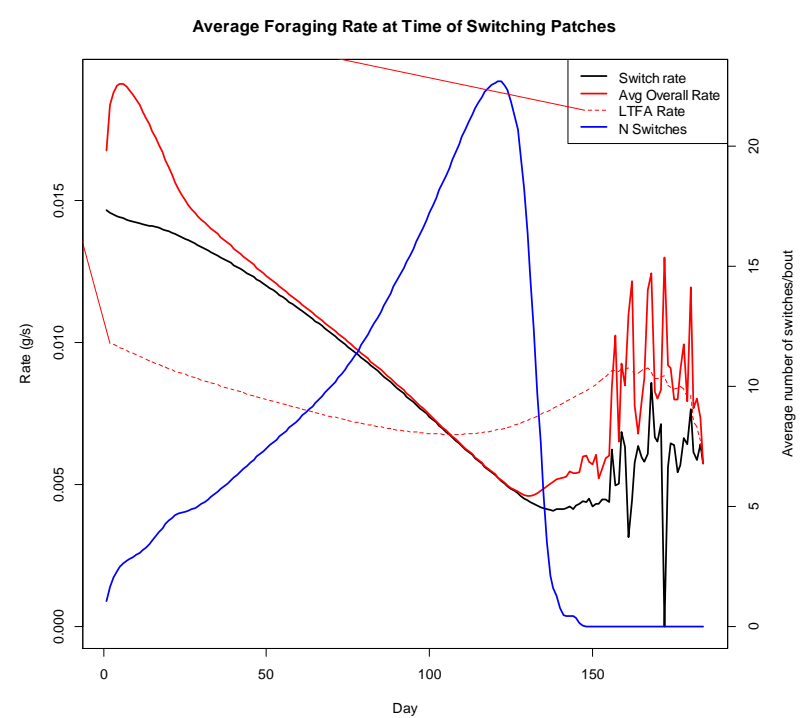

(b)

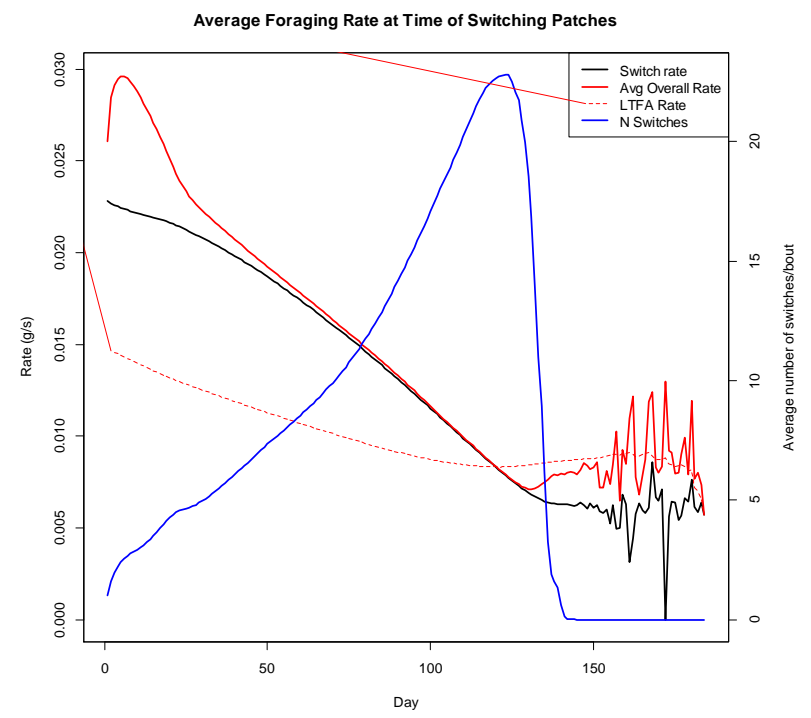

(d)

Figure B 2. Gain rate and MVT behavior for landscape with 2700 ha rice and 700 ha moist soil: (a) RLMVT, (b) OMVT, (c) XRLMVT, and (d) XOMVT. Solid red line is overall gain rate, dashed red line is LTFA rate, black line is switching threshold, and blue line (on right axis) is number of switches per bout. Note that scales differ between graphs. 


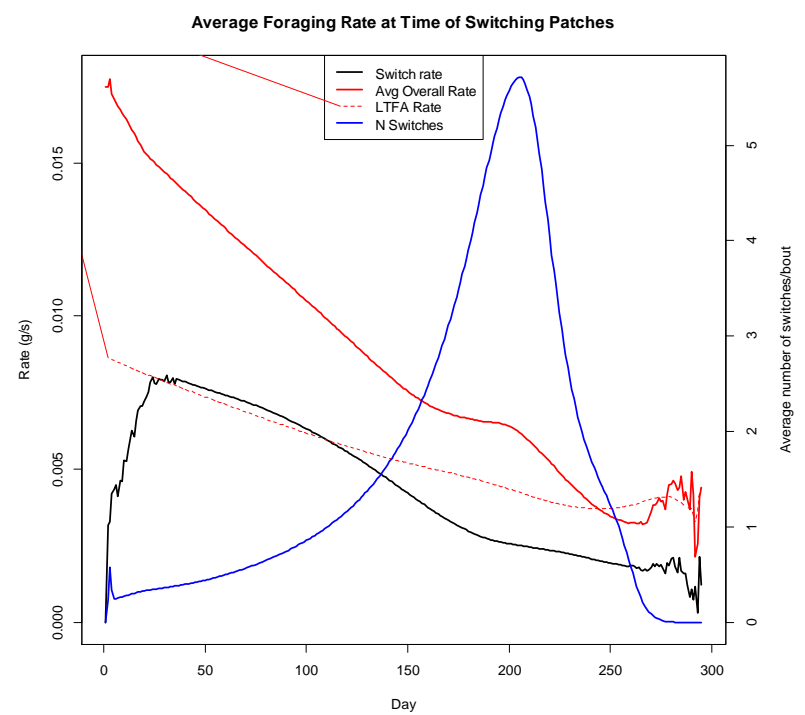

(a)

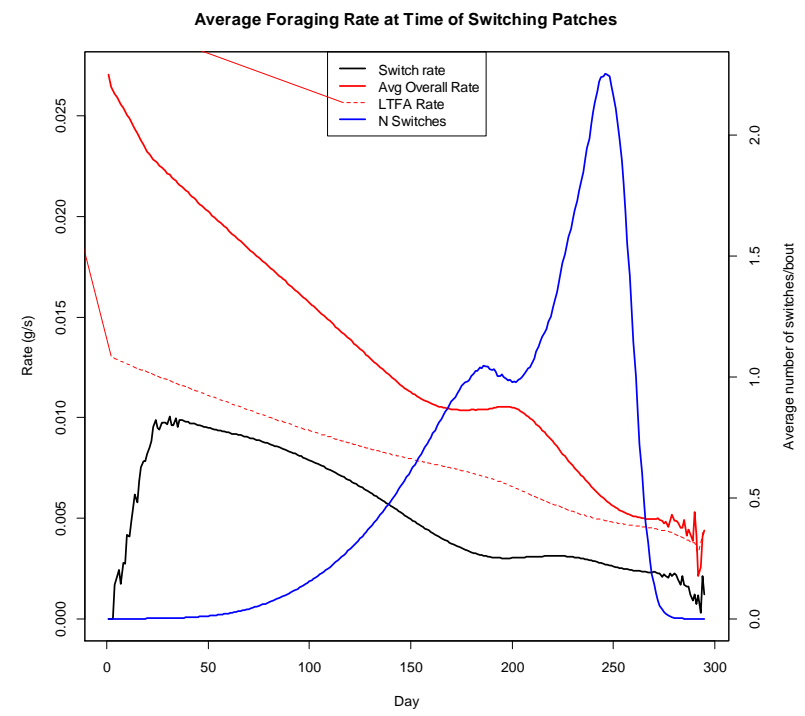

(c)

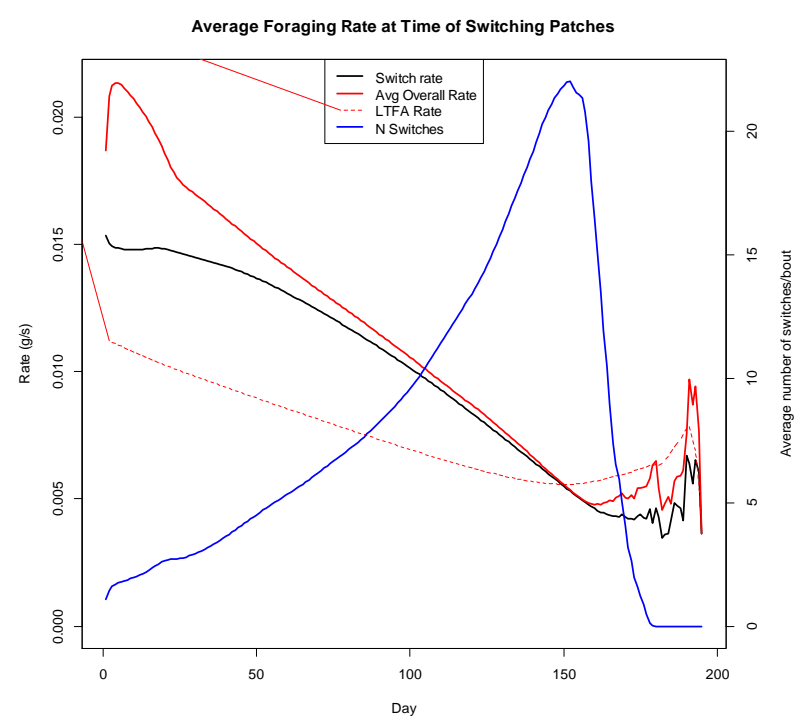

(b)

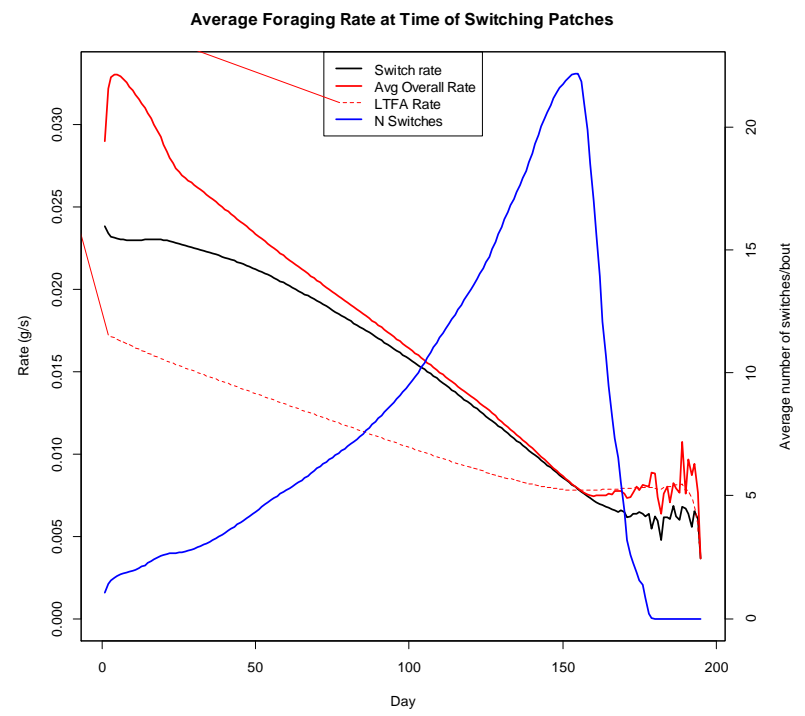

(d)

Figure B 3. Gain rate and MVT behavior for landscape with 2000 ha rice and 1400 ha moist soil: (a) RLMVT, (b) OMVT, (c) XRLMVT, and (d) XOMVT. Solid red line is overall gain rate, dashed red line is LTFA rate, black line is switching threshold, and blue line (on right axis) is number of switches per bout. Note that scales differ between graphs. 


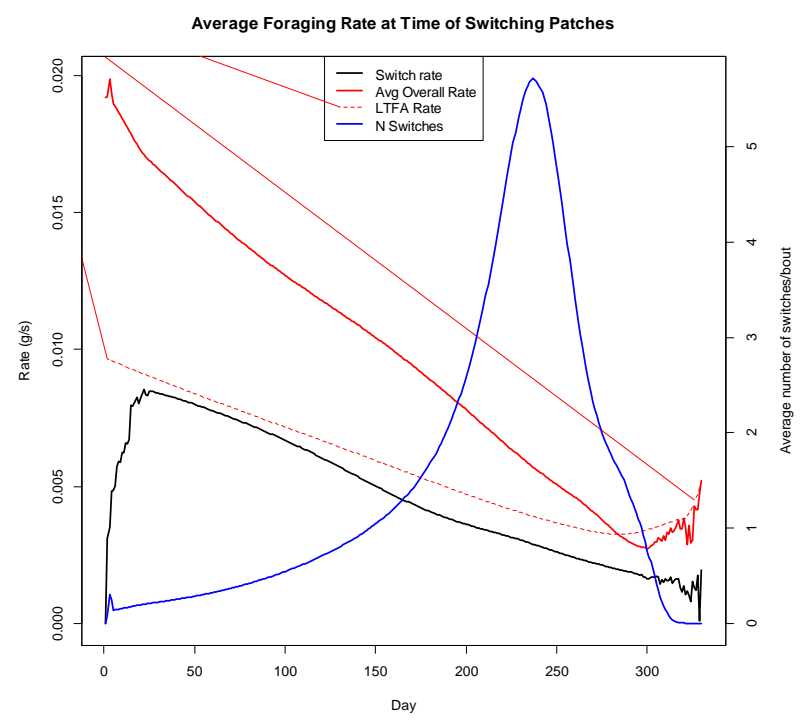

(a)

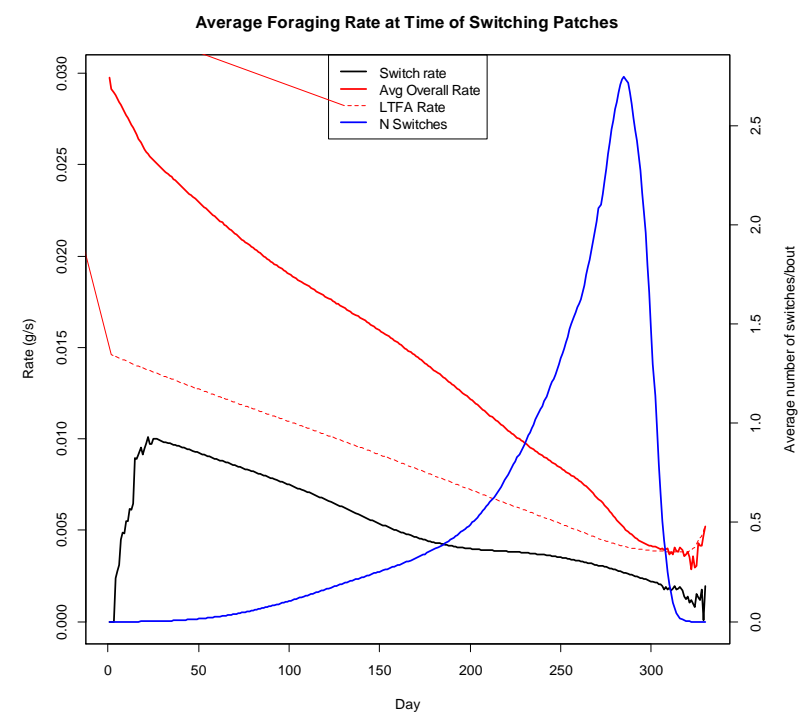

(c)

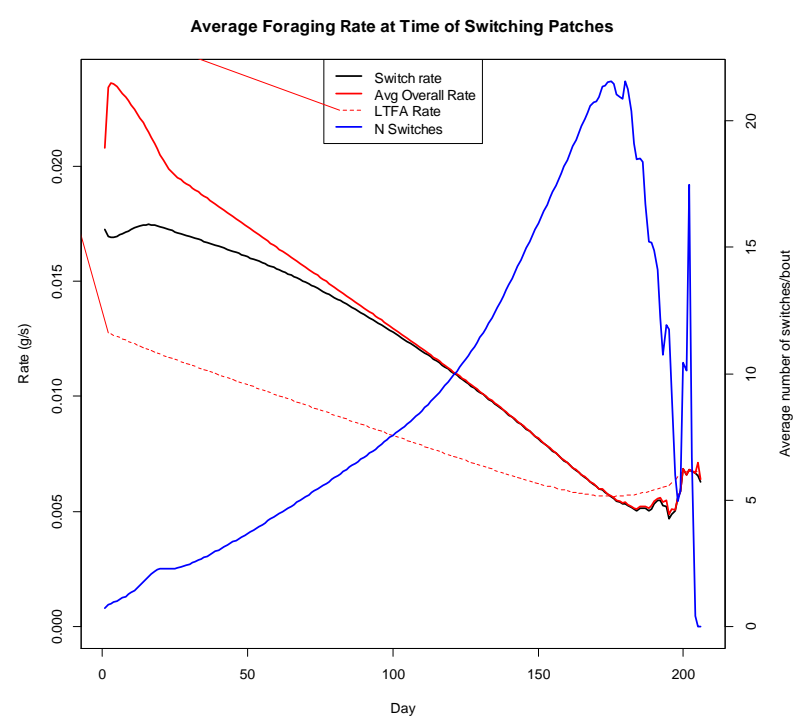

(b)

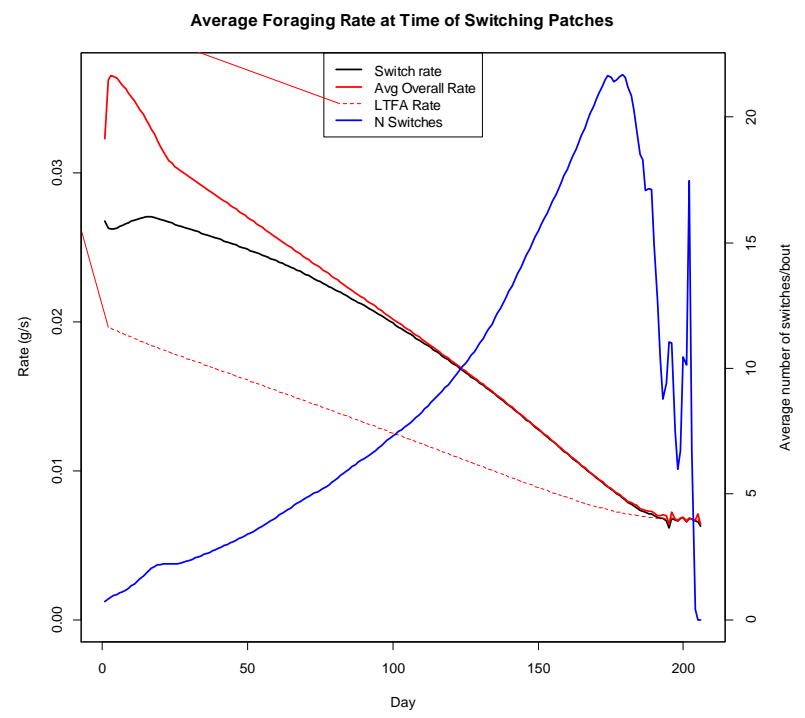

(d)

Figure B 4. Gain rate and MVT behavior for landscape with 1400 ha rice and 2000 ha moist soil (richest conditions): (a) RLMVT, (b) OMVT, (c) XRLMVT, and (d) XOMVT. Solid red line is overall gain rate, dashed red line is LTFA rate, black line is switching threshold, and blue line (on right axis) is number of switches per bout. Note that scales differ between graphs. 


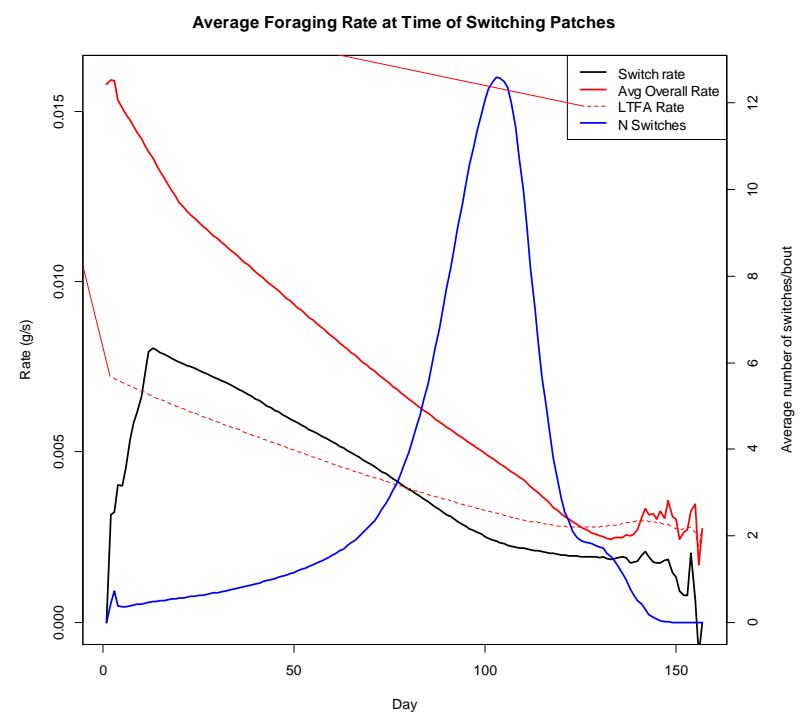

(a)

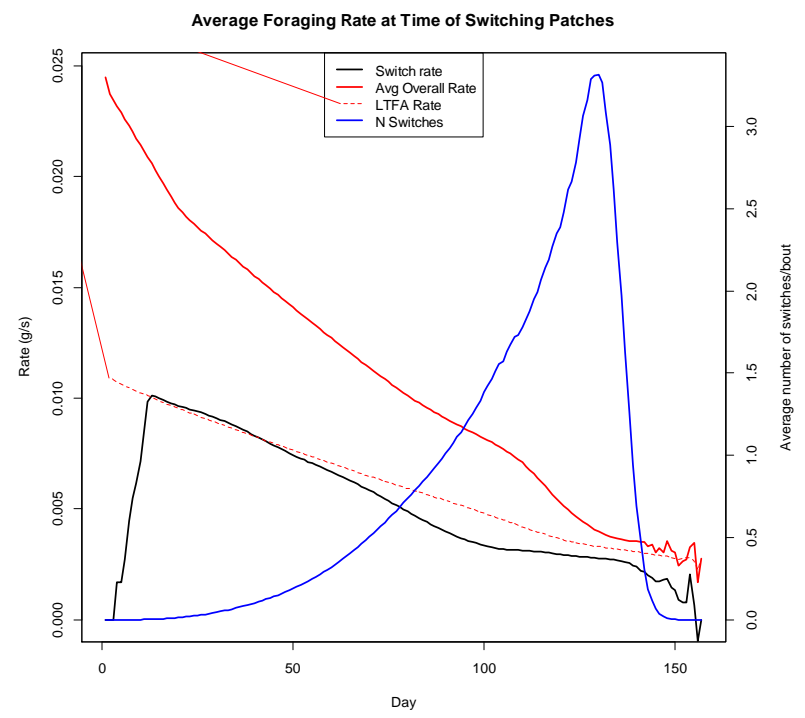

(c)

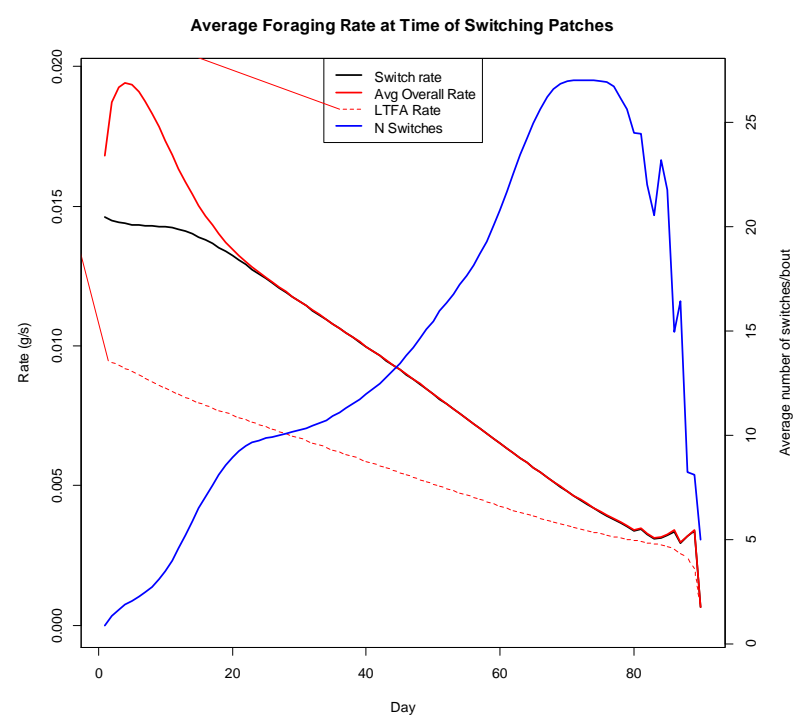

(b)

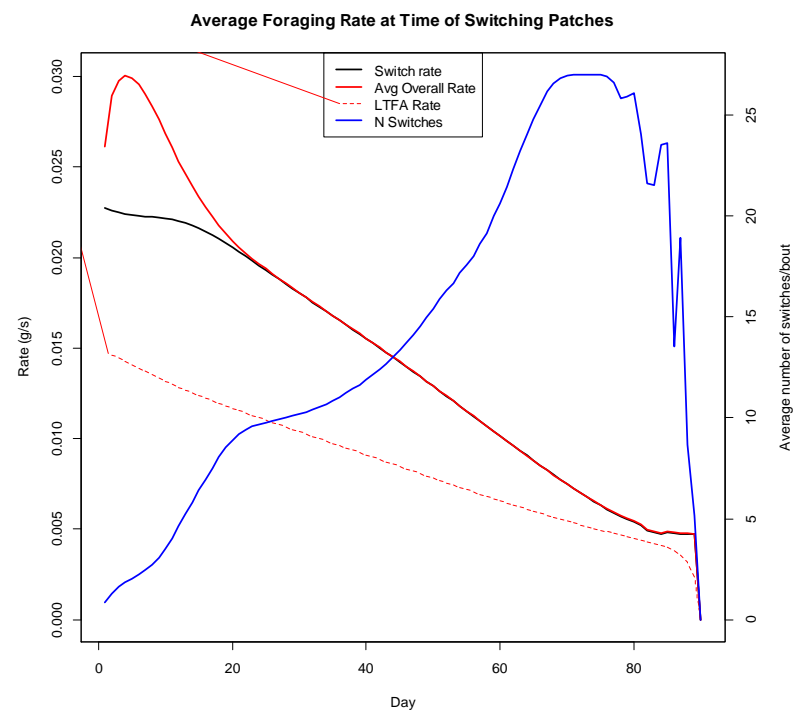

(d)

Figure B 5. Gain rate and MVT behavior for landscape with 1600 ha rice and 400 ha moist soil (poorest conditions): (a) RLMVT, (b) OMVT, (c) XRLMVT, and (d) XOMVT. Solid red line is overall gain rate, dashed red line is LTFA rate, black line is switching threshold, and blue line (on right axis) is number of switches per bout. Note that scales differ between graphs. 


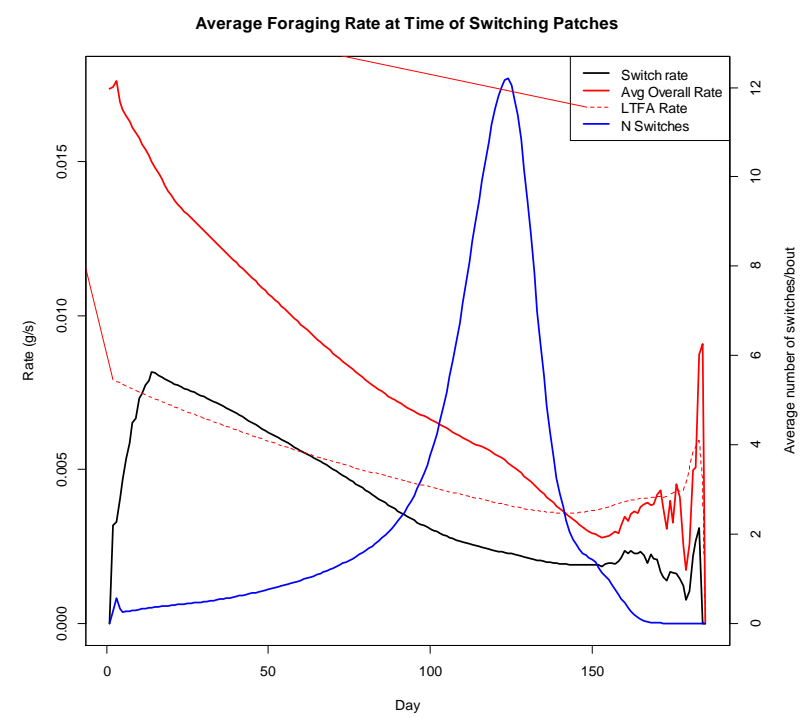

(a)

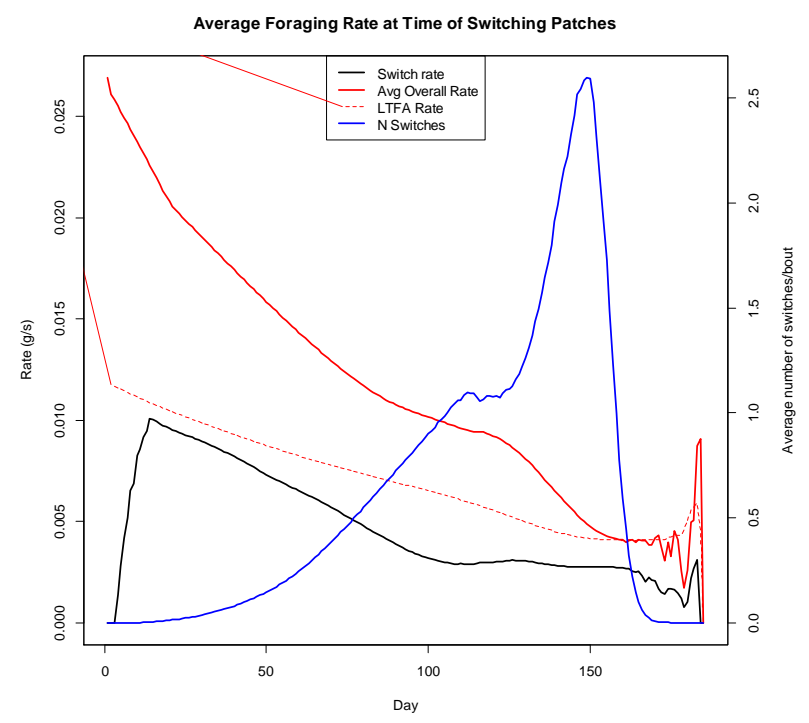

(c)

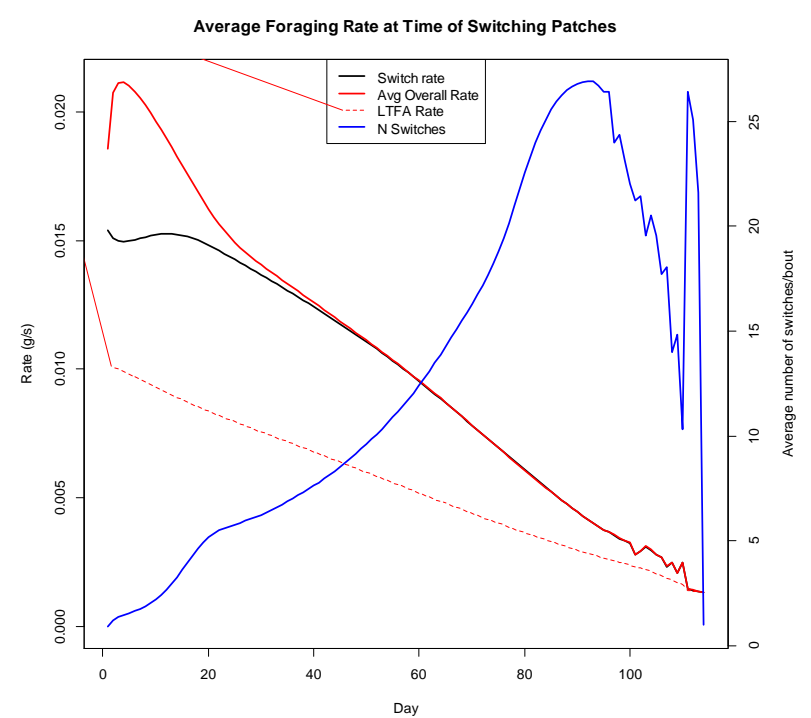

(b)

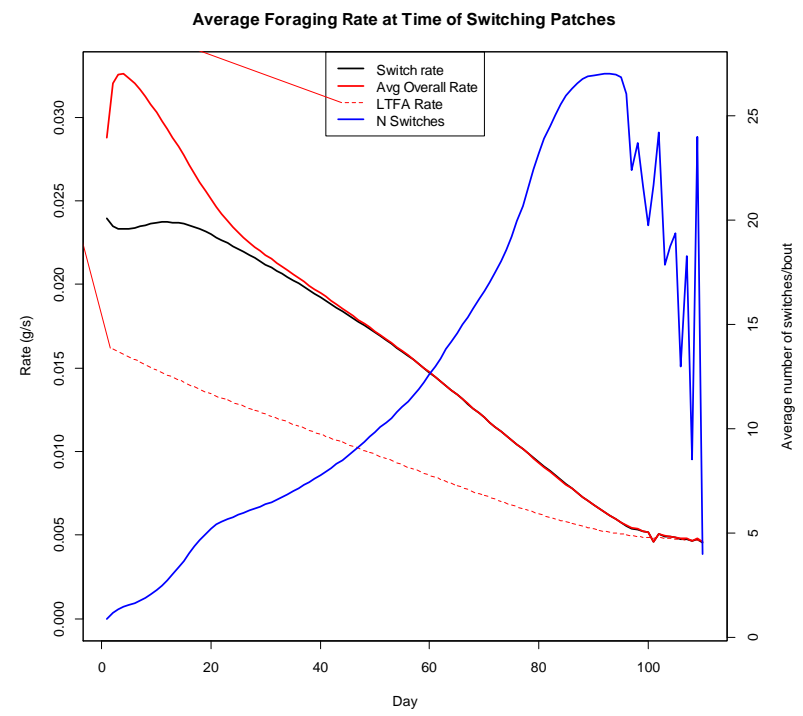

(d)

Figure B 6. Gain rate and MVT behavior for landscape with 1200 ha rice and 800 ha moist soil: (a) RLMVT, (b) OMVT, (c) XRLMVT, and (d) XOMVT. Solid red line is overall gain rate, dashed red line is LTFA rate, black line is switching threshold, and blue line (on right axis) is number of switches per bout. Note that scales differ between graphs. 


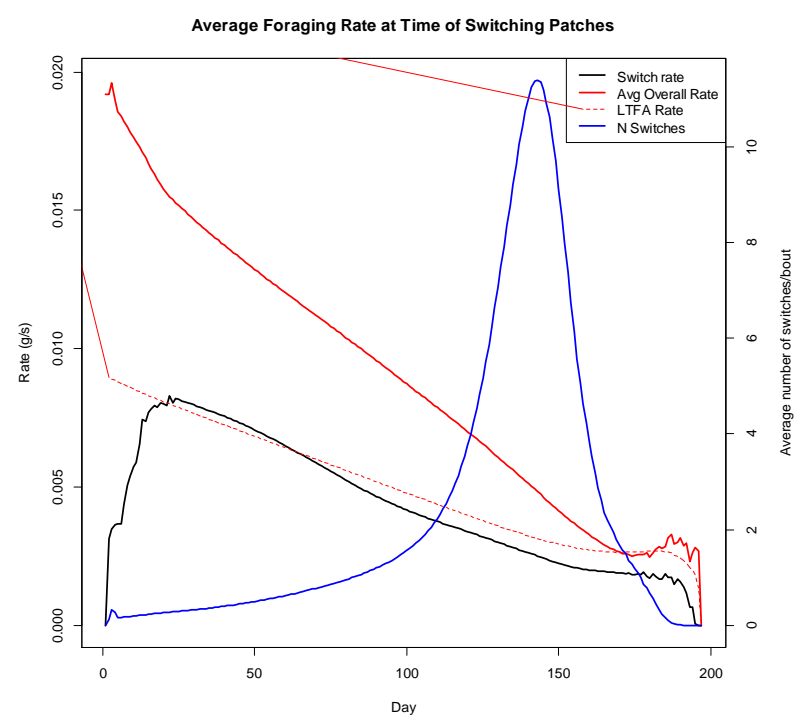

(a)

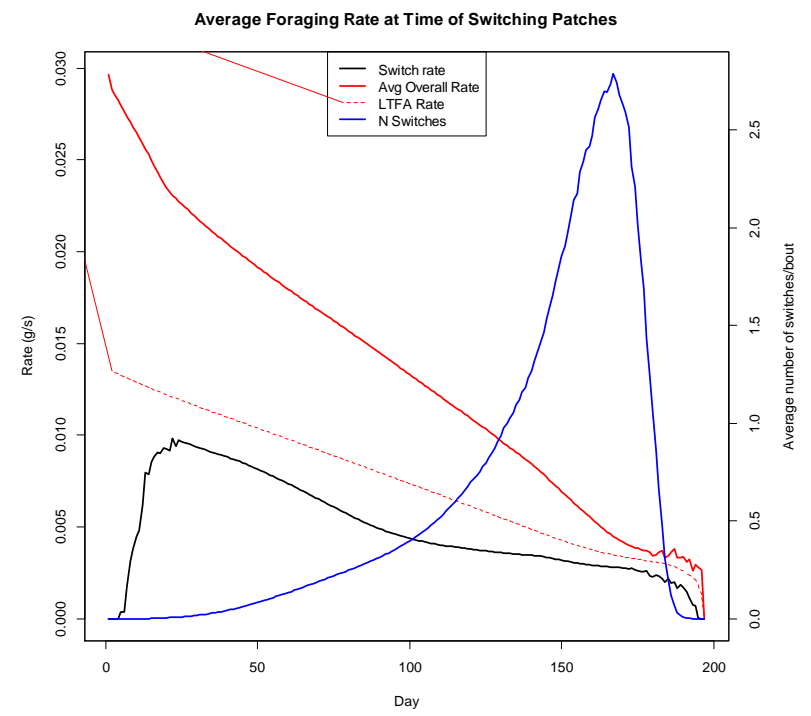

(c)

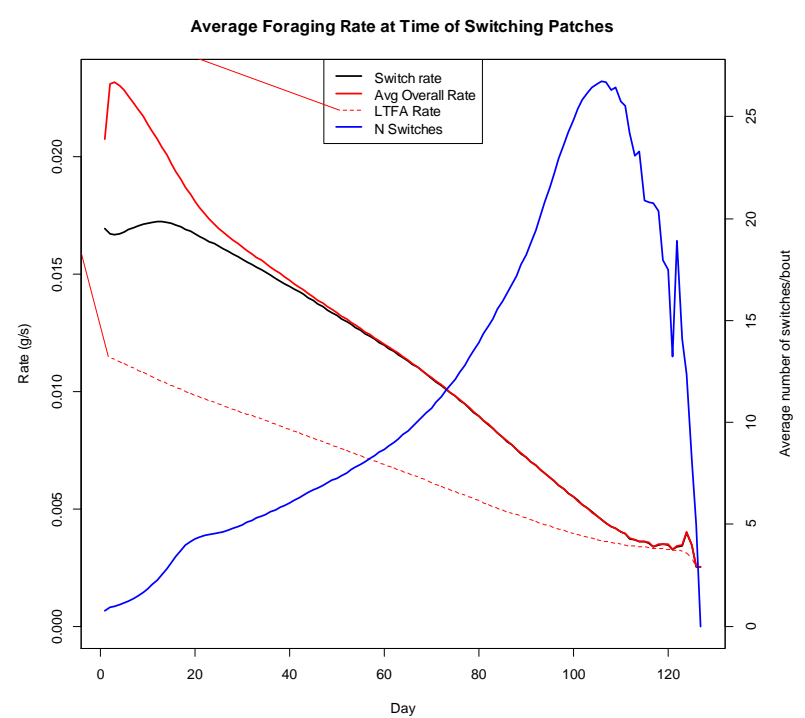

(b)

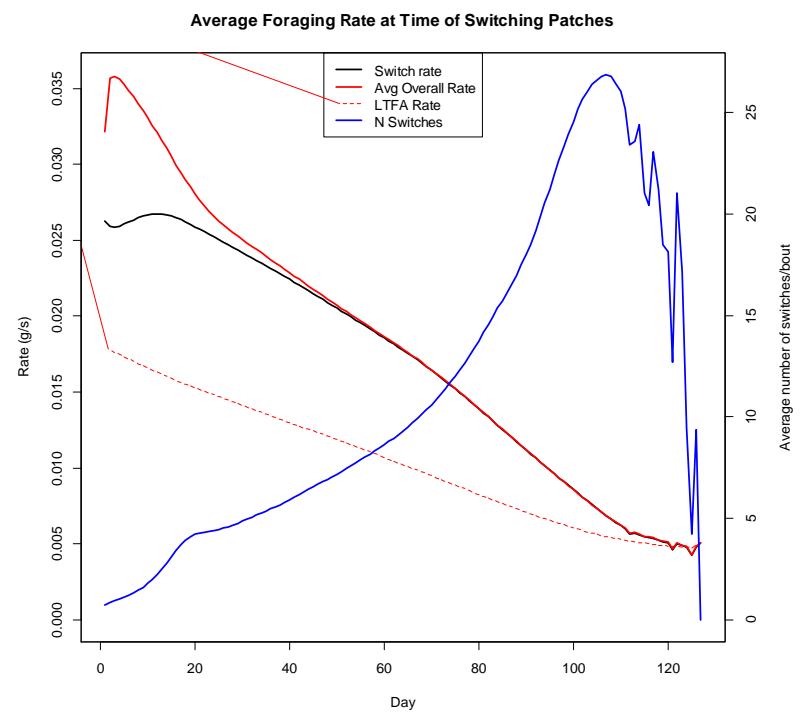

(d)

Figure B 7. Gain rate and MVT behavior for landscape with 800 ha rice and 1200 ha moist soil: (a) RLMVT, (b) OMVT, (c) XRLMVT, and (d) XOMVT. Solid red line is overall gain rate, dashed red line is LTFA rate, black line is switching threshold, and blue line (on right axis) is number of switches per bout. Note that scales differ between graphs. 


\section{Appendix C}

\section{$2 \quad$ C.1 Reinforcement Learning MVT}

Reinforcement learning MVT (RLMVT) is based on the Sutton and Barto (1998) n-

4 armed bandit, in which the problem of selecting an optimal behavior is conceived as playing a

5 slot machine with multiple $(n)$ levers. If each lever has a different probability of paying, the

6 optimum behavior is to always select the lever with the highest probability. After accumulating a

7 sufficient number of trials, an agent that uses reinforcement learning would only select that lever.

8 This can be simulated in the following way. If the arms are numbered from 1 to $n$, the action of

9 pulling a given arm $a$ will stochastically result in reward, $r$, between 0 and the upper limit of

10 possible rewards. Over a given number of trials for each arm, $z_{a}$, the agent can build up an

11 expected reward for each action

$$
R_{a}=\frac{\sum_{z=0}^{z_{a}} r_{z}}{z_{a}}
$$

12 As $z_{a}$ approaches infinity, $R_{a}$ will approach the true expected reward for arm $a$. Thus, the optimal

13 choice of arm is achieved for $a$ where $R_{a}$ is maximum. Of course, in the short-term, this approach

14 has difficulties: preceding $z=0$ there is no maximum and as $z_{a}$ increases there is no way for the

15 agent to know when it has enough data to ensure that values of $R_{a}$ are "close enough" to the true

16 values. Thus, Sutton and Barto recommend an $\varepsilon$-greedy strategy in which the agent randomly

17 samples actions other than the apparent current optimum in some small proportion of cases $\varepsilon$.

18 Though the $\varepsilon$-greedy strategy is reasonably effective, Sutton and Barto (1998) note that it

19 suffers from an inefficiency in that alternate strategies are selected uniformly at random; that is,

20 the apparent worst action is as likely to be sampled as the apparent second best action. They

21 therefore offer an improved version they refer to as softmax in which the likelihood of a given

22 action $a$ is weighted according to the current estimate of $R_{a}$. In particular, they suggest a

23 weighting based on the Boltzmann distribution in which the probability $(\mathrm{P}(a))$ of action $a$ is 


$$
\mathrm{P}(a)=\frac{e^{R_{a} / \tau}}{\sum_{b=1}^{n} e^{R_{b} / \tau}}
$$

1 in which $\tau$ is a positive number referred to as temperature, low values of which approach $\varepsilon$ -

2 greedy behavior and high values of which decrease the effect of expected reward (that is, as $\tau$

3 approaches infinity, all actions become equally likely regardless of expected reward; see

4 Appendix A for a graphical representation for the parameters used in this research).

As discussed in the main text, applying reinforcement learning to MVT requires mapping

6 switching thresholds to the arms of the $n$-armed slot machine. Each threshold is represented by a

7 lever $a$, corresponding to the net gain across all patches using the given switching threshold as

8 the reward for that arm. We then find the arm, or action, that embodies the maximum gain, and

9 thus the optimal rate at which to switch patches under MVT.

We follow Wawerla and Vaughan (2009) in transforming the continuous range of possible

11 switching thresholds $(\theta)$ equally into $n$ bins, from the expected minimum gain rate $\left(\theta_{\min }\right)$ to the

12 expected maximum gain rate $\left(\theta_{\max }\right)$. These bins map on to the discrete arms on an $n$-armed bandit.

13 Each bin's center $\left(\Theta_{a}\right)$ is used as the switching threshold $\theta$ when action $a$ is selected. The

14 estimated reward for action $a, R_{a}$ (analogous to $R_{a}$ in Equation $\mathrm{C} 1$ ) is a weighted rolling average

15 of the gain rate $\mu$ achieved using the switching rate $\theta_{a}$ :

$$
\mu=\frac{f}{t^{\prime}+t}
$$

16 where $f$ was the amount of food accumulated in the patch, $t$ ' was the amount of time spent

17 traveling between patches, and $t$ was the amount of time spent in the patch. The estimated reward

18 is updated at time step $s$ by

$$
R_{a_{s}}=R_{a_{s-1}}+d_{\text {reward }}\left(\mu-R_{a_{s-1}}\right),
$$

19 where $d_{\text {reward }}$ is the weight of the most recent gain rate's contribution to the rolling average; at

20 time step 0 , the estimated reward for all bins is 0.

21 Of course, none of the equally-spaced bin centers are likely to coincide with the actual 
1 optimum switching threshold. To address this issue, Wawerla and Vaughan (2009) allowed the

2 bin centers to be optimized by following a random walk weighted by the most recent gain rate $\mu$.

3 So, at each time step instead of using $\theta=\Theta_{a}$, the actual threshold chosen was drawn uniformly

4 randomly from

5 where $\delta$ is the initial width of the bins

$$
\left[\Theta_{a}-k \delta, \Theta_{a}+k \delta\right]
$$

$$
\delta=\frac{\theta_{\max }-\theta_{\min }}{n},
$$

6 and $k$ is a factor controlling the size of the random walk. This value for $\theta$ is then allowed to

7 update the value for $\Theta_{a}$, weighted by the achieved gain rate:

$$
\Theta_{a_{s}}=\frac{R_{a_{s-1}} \cdot \Theta_{a_{s-1}}+\mu \cdot \theta}{R_{a_{s-1}}+\mu \cdot \theta} .
$$

8 In this way, bin centers moved slowly to optimal values.

9 Since at time $s=0$ there is no data for any of the bins, we initialize the bins during the first

$10 n$ time steps by setting bin centers $\Theta_{a}$ (and thus switching threshold $\theta$ ) strictly to initial bin center

$$
\Theta_{a}=\theta=s \delta+\theta_{\min }-\frac{\delta}{2}
$$

11 (a slight modification of Wawerla \& Vaughan, 2009; J. Wawerla, personal communication,

12 August 23, 2012). Likewise, during the first $n$ time steps, expected reward is set directly to the

13 achieved gain rate, $R_{a}=\mu$.

14 Once the bins were initialized, Wawerla and Vaughan (2009) used Sutton and Barto's

15 (1998) softmax algorithm to select the bin $a$ from which to draw the switching threshold, with

16 probability as given in Equation C2.

\section{$17 \quad$ C.2 Online MVT}

18 In developing a method for continuous estimating of the proper switching threshold,

19 Wawerla and Vaughan (2010) note that in classical MVT the cost of travel between patches

$20 t^{\prime} E_{\text {travel }}$ is not affected by changes in how the forager makes patch-switching decisions (main text, 
1 Equation 3), so they drop that term to allow maximization of

$$
\frac{\sum_{p} \pi_{p} g_{p}\left(T_{p}\right)}{t^{\prime}+\sum_{p} \pi_{p} T_{p}}
$$

2 (though see main text discussion for caveats about omitting energetic cost of travel). Furthermore,

3 the authors once again eliminate the concept of patch types since patches vary continuously in

4 resource availability. Then Equation C9 must be rewritten with $N$ equal to the total number of

5 patches (for gross average gain rate) as

$$
\frac{\frac{1}{N} \sum_{p=1}^{N} g_{p}\left(T_{p}\right)}{t^{\prime}+\frac{1}{N} \sum_{p=1}^{N} T_{p}}
$$

6 If the forager is in the process of foraging and has arrived at patch $p$ in its journey, then this can

7 be calculated by

$$
\widehat{M}_{p}=\frac{g_{p}\left(T_{p}\right)+G}{T_{p}+\widehat{t}^{\prime}+T}
$$
where $G$ is the sum of gain in the previous patches, $T$ is the sum of time in the previous patches,

9 and $\widehat{M}_{p}$ is the estimate for the long-term gain rate while in patch $p$. The agent also estimates $t^{\prime}$,

10 average inter-patch travel time, since it cannot know the true value for the whole landscape based

11 only on its own, limited experience. At each time step $s$, the current estimate of the average

12 switching time, $\widehat{t}_{s}^{\prime}$, is updated by using a weighted rolling average of inter-patch travel times as

$$
\widehat{t}_{s}^{\prime}=\widehat{t}_{s-1}^{\prime}+d_{\text {travel }}\left(t_{p}^{\prime}-\widehat{t}_{s-1}^{\prime}\right) \text {, }
$$

13 where $t_{p}^{\prime}$ is the travel time from the previous patch to patch $p$ and $d_{\text {travel }}$ is the weight of the

14 current travel time's contribution to the rolling average. The current estimate is used for $\widehat{t^{\prime}}$ in

15 Equation C11. Because the gain in each patch decreases across time as resources are depleted,

16 the long-term gain rate may fall below the previously estimated long-term gain rate when the

17 current patch's contribution to that estimate falls low enough, if that in fact occurs during a

18 foraging bout (the forager may reach its daily intake limit or time limit long before that occurs).

19 We detect this by subtracting the value at $s$ from the value at $s-1$ and switching patches when the 
1 value is less than or equal to zero. 